DESY 15-113

FNT/2015/01

\title{
Automation of electroweak corrections for LHC processes
}

\author{
Mauro Chiesa \\ INFN, Sezione di Pavia, Via A. Bassi 6, 27100 Pavia, Italy \\ Nicolas Greiner \\ DESY Theory Group, Notkestr. 85, D-22607 Hamburg, Germany \\ Francesco Tramontano \\ Dipartimento di Fisica, Università di Napoli "Federico II" \\ and INFN, sezione di Napoli, via Cintia, 80126 Napoli, Italy
}

\begin{abstract}
For the Run 2 of the LHC next-to-leading order electroweak corrections will play an important role. Even though they are typically moderate at the level of total cross sections they can lead to substantial deviations in the shapes of distributions. In particular for new physics searches but also for a precise determination of Standard Model observables their inclusion in the theoretical predictions is mandatory for a reliable estimation of the Standard Model contribution. In this article we review the status and recent developments in electroweak calculations and their automation for LHC processes. We discuss general issues and properties of NLO electroweak corrections and present some examples, including the full calculation of the NLO corrections to the production of a $W$-boson in association with two jets computed using GoSAm interfaced to MADDipole.
\end{abstract}

PACS: 11.15.Bt, 12.15.Lk, 13.85.-t

\section{Contents}

\section{Introduction}

A. Physical motivations

B. Electroweak corrections for the LHC: quest for automation 
II. Virtual corrections and scheme dependence

A. Electroweak renormalization and counterterms

B. Electroweak insertion operator

C. Subtraction terms and scheme dependence

D. Examples
1. $Z \rightarrow \nu \bar{\nu}$
2. $W^{+} \rightarrow u \bar{d}$
3. $u \bar{u} \rightarrow c \bar{c}$

III. Real emission and subtraction procedures

IV. Electroweak corrections to $W^{+}$plus two jets

A. Computational setup

1. Virtual corrections

2. Regularization scheme dependence

3. Real emission

4. Parameters

B. Numerical Results

C. Virtual amplitudes for single phase space point

1. $\bar{u} u \rightarrow W^{+} \bar{u} d$

2. $u \bar{d} \rightarrow W^{+} c \bar{c}$

3. $u \bar{d} \rightarrow W^{+} s \bar{s}$

4. $u \bar{d} \rightarrow W^{+} g g$

5. $\bar{u} u \rightarrow W^{+} \bar{c} s$

6. $\bar{d} d \rightarrow W^{+} \bar{c} s$

7. $u \bar{d} \rightarrow W^{+} d \bar{d}$

\section{Conclusions}

\section{Acknowledgements}

\section{References}




\section{INTRODUCTION}

The Large Hadron Collider (LHC) was designed to provide a deeper insight into the microscopic world and increase our knowledge about the fundamental interactions occurring in nature.

The recent discovery of the Higgs boson [1, 2] supports the predictions made by the Standard Model of particle physics [3 7]. It is not yet clear though, whether the discovered boson is exactly the one predicted by the Standard Model, or whether it originates from supersymmetric theories, or whether it is something completely different mimicking the role and the properties of a Higgs boson.

There is the hope that the run 2 at the LHC will show not only more phenomenological properties of the Higgs particle, but also additional new physics beyond the Standard Model (BSM). As in principle it is not known how new physics will manifest itself, one has to look for even small deviations from the Standard Model predictions. Completely different new physics scenarios can lead to very similar predictions, which makes it difficult to distinguish among the models. Besides precise experimental measurements this requires precise theoretical predictions.

At high energy hadron colliders, final states typically involve hundreds of particles (hadrons, leptons, neutrinos and photons). General purpose Monte Carlo (MC) event generators like HERWIG [8], HERWIG++ [9], PYTHIA 6 [10], PYTHIA 8 [11] and SHERPA [12] can provide a fully exclusive description of such complicated reactions. These tools perform several tasks ranging from the computation of hard scattering processes to the simulation of multiple soft and/or collinear QCD and QED radiation by means of parton shower algorithms. MC event generators also provide a description of the non-perturbative effects like hadronization and formation of the underlying event. General purpose MC generators play a crucial role in collider physics and indeed there is no experimental analysis or realistic phenomenological study which does not rely at least in part on these codes.

One of the drawbacks of general purpose MC event generators is the fact that hard scattering processes (and, as a consequence, overall normalizations) are computed at tree-level accuracy, while precise theoretical predictions for several processes of phenomenological interest require at least the calculation of next-to-leading order (NLO) contributions in perturbation theory. Typically, at hadron colliders the largest contributions come from QCD corrections. In the last recent years, lots of progress has been made in calculating NLO QCD corrections: new 
algorithms, such as the ones based on the OPP method [13 15] or on the generalized unitarity techniques [16 18], have been proposed to compute one loop virtual amplitudes numerically or semi-analytically, i.e. bypassing the explicit calculation of one loop Feynman diagrams from the Feynman rules of the theory. In particular, a lot of effort has been put into the automation of such intricate and time consuming calculations and several tools have been developed either based on generalized unitarity methods, such as BLACKHAT [19] and NJET [20], or on OPP-inspired techniques, such as GoSam [21, 22], HELAC-NLO 23], MAdLoop [24]/ MadGraph [25, 26]. In codes like OpenLoops [27] and RECOLA [28] one loop amplitudes are computed numerically from tree level amplitudes by means of recursion relations applied to Feynman diagrams and off-shell currents, respectively. The fact that calculations were not carried out any more on a process-by-process basis but obtained by automating the underlying building blocks and interfacing them in a standardized way, played a major role in what has become known as the NLO revolution.

The NLO revolution of QCD corrections set a new standard for the data/theory comparison at run I of the LHC. While, at the TEVATRON, data were typically compared to the predictions of LO multi-parton event generators such as ALPGEN [29], SHERPA and MADGRAPH that were used to generate samples with different hard parton multiplicities merged to parton showers in the CKKW [30, 31] or MLM [32] framework, the standard theoretical benchmark at the run I of the LHC for most of the analyses are NLO QCD computations consistently matched with parton showers within the POWHEG [33 35] or the MC@NLO [36] framework. Methods like FxFx [37], MEPS@NLO [38, 39], MiNLO [40, 41] and UNLOPS [42] were also developed to merge parton showers and NLO samples with different parton multiplicity. Both matched and merged calculations rely on MC event generators like HERWIG, PYTHIA and SHERPA for what concerns showering and non perturbative effects (for the description of multiple photon radiation off final state leptons the code PHOTOS [43] is also widely used). Needless to say, this NLO revolution pushed the frontier of fixed order QCD corrections to the NNLO level of accuracy.

A lot of work has been done in the field of automation of one loop QCD corrections. However, as we will point out in the next paragraphs, in view of the $14 \mathrm{TeV}$ run of the LHC there are the physical motivations to extend the technical know-how of these automated approaches also to the context of electroweak corrections. Because of this point, we decided to focus the present 
review on the practical issues that one has to face in order to build a tool for the automated computation of NLO electroweak corrections to LHC processes starting from already available codes developed for the computation of the different building blocks of an NLO calculation. In particular, we will show how this task can be accomplished in GoSAM+MADDipoLE [4446] framework for the computation of the relevant amplitudes and the subtraction of infrared singularities respectively, once the effect of electroweak renormalization has been properly taken into account.

The paper is organized as follows. In subsection IA we give a short overview about the phenomenological motivations for the calculation of NLO electroweak corrections at high energy hadron colliders. In this context, in subsection โB we explain the main reasons why the automation of $\mathcal{O}(\alpha)$ corrections will play a crucial role at the $14 \mathrm{TeV}$ run of the LHC and, of course, at future colliders. We summarize the state of the art for the automation of NLO electroweak corrections, describing very briefly the existing tools and the phenomenological studies obtained with such codes. As stated above, with the present review we want to show how the automation of NLO electroweak corrections can be obtained starting from already existing tools: in particular we work in the GoSAM+MADDIPOLE framework. In section Iwe discuss some of the issues related to the computation of virtual one loop electroweak corrections, such as the UV and IR regularization scheme dependence, the inclusion of electroweak renormalization and the subtraction of infrared singularities. As codes like GoSAm work in dimensional reduction (DRED), while electroweak renormalization counterterms and infrared subtraction terms are usually derived in conventional dimensional regularization (CDR), we give the transition rules from CDR to DRED regularization schemes for both renormalization counterterms and infrared subtraction terms. We show how these scheme conversion works with a few simple examples, checking explicitly the regularization scheme independence of the sum of virtual one loop corrections, renormalization counterterms and integrated dipoles. Real radiation effects, and in particular the treatment of photon radiation off massless fermions, are described in section [II. As a realistic example of the technical issues described in sections [I] and III, we describe in detail a calculation of the one loop electroweak corrections to the process $p p \rightarrow W^{+} j j$ in the GoSAM+MADDipole framework in section IV] We show the results of some internal consistency checks concerning poles cancellation and we briefly discuss the phenomenological impact of the electroweak corrections to the $W$ production in association with 
2 jets.

\section{A. Physical motivations}

In the next run of the LHC, with higher center of mass energy and also higher luminosity, electroweak radiative corrections will play a more and more important role.

First of all, the high luminosity will allow to perform electroweak precision measurements. This is for example the case for the $W$ boson mass measurements in the charged Drell-Yan process, where $M_{W}$ is determined indirectly from a template fit of the distributions of transverse mass $M_{T}=\sqrt{2 p_{T}^{l} p_{T}^{\nu}\left(1-\cos \Delta \phi_{\mathrm{l} \nu}\right)}$ of the lepton neutrino system, of the the lepton $p_{T}$ and of the missing transverse energy. It is well known from the TEVATRON experience that a precise knowledge of the electroweak radiative corrections to the Drell-Yan is mandatory, as they alter the shapes of the above-mentioned distributions and thus affect the $W$ mass determination. This is the reason why different groups spent a lot of effort to compute the one loop electroweak corrections to the Drell-Yan and several simulation codes have been provided, such as HORACE [47, 48], RADY [49 51], SANC [52, 53], WGRAD [54], WINHAC [55, 56]

and ZGRAD [57]. All of these codes have LO QCD accuracy and NLO EW accuracy. In particular SANC also include NLO QCD corrections, while HORACE includes the effect of all order photon radiation properly matched to the $\mathcal{O}(\alpha)$ corrections. NLO EW corrections are added to the $\mathcal{O}\left(\alpha_{S}^{2}\right)$ ones in the FEWZ code [58], while factorized NLO EW and NLO QCD corrections to the single $W$ and $Z$ production matched with QED and QCD parton shower have been implemented in the POWHEG-BOX Monte Carlo event generator [59 61].

The higher luminosity will also improve the experimental accuracy for several other processes besides the Drell-Yan process. In order to match the foreseen experimental precision, theoretical predictions for many processes should include at least the effect of $\mathcal{O}(\alpha)$ corrections, as can be seen for example by looking at the Les Houches wish-list in Tables 1-3 of Ref. [62]. It is interesting to noting how the Les Houches wish-list also points out that, in order to fully exploit the physics potential of the LHC, fixed order EW computations should be properly matched with parton showers. At present, the issue of matching NLO EW corrections with parton showers has only been addressed for Drell-Yan in Refs. [59, 61] and very recently for Higgs decay into four leptons in Ref. [63]. 
In the context of the high precision determination of the Standard Model parameters such as, for example, the $W$ boson mass, the theoretical uncertainties related to parton distribution functions (PDFs) will eventually become a limitation. Recently new PDF sets [64 67] have been released which include the constraints coming from LHC run I data. PDFs are obtained by fitting experimental data with theoretical computations that are performed at the highest available perturbative order which at present is typically NNLO QCD. In Ref. [65] NLO electroweak corrections have been considered in the fit of Drell-Yan data [68]: the systematic inclusion of one loop EW corrections in PDF fits will move in the direction of reducing further the theoretical uncertainty related to PDFs. Besides the impact of EW corrections on PDF fits, electroweak corrections also affect the description of PDF evolution: QED corrections, for instance, can be included in the evolution equations, leading to the definition of the photon PDF 69 73]. In turn, the knowledge of the photon PDF allows to compute the so-called photon induced processes (i.e. partonic channels with photons in the initial state) which should be consistently included in the computation of $\mathcal{O}(\alpha)$ corrections[242]. At variance with the case of QED, the effect of weak corrections to PDF evolution [74, 75] has still to be investigated.

If on one hand the higher luminosity will improve the experimental accuracy, on the other hand the increase of the center of mass energy will allow to probe more and more extreme regions of the phase space, such as the ones characterized by a large jet multiplicity at high $p_{T}$ and missing transverse energy (also with additional hard isolated leptons) or by the production of boosted objects in association with jets, that are of great interest for both the direct new physics searches and also for quite challenging Standard Model measurements (see for instance the results of Refs. [76 81] for $W+$ multi-jet and $Z+$ multi-jet production, as possible examples of direct new physics searches at the LHC we refer to [82 85]). In these extreme kinematical configurations one loop electroweak corrections are dominated by double and single logarithms of the typical energy scale of the process over the gauge boson masses (the so called Sudakov logarithms [86, 87]). The Sudakov logarithms basically correspond to the infrared regime of the virtual one loop weak corrections, where the gauge boson masses can be neglected if compared to the other energy scales involved. In the Sudakov limit the weak boson masses play the role of physical regulators for the infrared singularities of the loop diagrams, which in turn manifest themselves as logarithms of the energy scale over the infrared cutoffs $M_{W}$ and $M_{Z}$ [88 96]. The Sudakov corrections are negative and usually have a moderate impact on the total 
cross sections. However these corrections are sizable in particular in the tails of the $p_{T}$ or $H_{T}$ distributions [62, 97] where they can be of the order of several tens of percent $(-40 \%,-50 \%)$ at the $14 \mathrm{TeV}$ LHC and become even larger at the center of mass energies of the proposed future hadron colliders such as the High-Energy LHC or the hh-FCC [98, 99]. These large negative effects should be included in the theoretical predictions and are a clear indication of the role that the $\mathcal{O}(\alpha)$ corrections will play at high energy hadron colliders (also for processes with many final state particles).

\section{B. Electroweak corrections for the LHC: quest for automation}

At present full one loop electroweak corrections are available only for a limited class of LHC processes, such as charged and neutral Drell-Yan, $V+1$ jet $(V=Z, W, \gamma)$ 100 103], dilepton+jets [104 106], single top [107 109], $t \bar{t}[110$ 116], dijet [117 119], $Z / W+H$ [120, 121] (including the $Z / W$ decay products), $H$ production in vector boson fusion [122, 123], $V V^{\prime}$ (with on-shell vector bosons or in pole approximation) [124 129], $W W+1$ jet [130], $W Z Z$ [131], $W W Z$ [132], $H \rightarrow 4 f$ [63, 133], $W \gamma$ production [134], $t \bar{t}+H$ [135, 136]. More recently, exact $\mathcal{O}(\alpha)$ corrections to $Z(\rightarrow \bar{l})+2$ jets [137], $W+n$ jets $(n \leq 3)$ [138] and $t \bar{t}+H / Z / W$ [135, 139]. Except for the last three examples, all the above computations have been carried out on a process-specific approach and are basically limited to processes with four or at most five external particles: the main reason of this limitation is the fact that the computational complexity grows considerably as the number of external leg particles increases.

As already stressed in the previous paragraphs, in order to fully exploit the physics potential of the second run of the LHC, the computation of the NLO electroweak corrections will be mandatory for a very large number of processes (also with large final state multiplicities). It should be clear that in this context a process-specific approach for NLO EW calculations is no longer feasible and more general and process-independent techniques should be developed.

As far as only the Sudakov regime is concerned, the universality of the infrared limit of weak corrections can be exploited to develop general algorithms for the calculation of the EW corrections in the logarithmic approximation [93, 95, 96, 140]. Following this approach, the Sudakov corrections to diboson [124, 141 143], vector boson plus multi-jets [97, 144], $t \bar{t}+$ jets [145], $H$ [146, 147] and $H+$ jet [144] production have been computed pointing out further the phe- 
nomenological impact of the EW corrections at high energies. Order $\alpha$ corrections to dijet, Drell-Yan and $t \bar{t}$ production have been recently included in the MCFM Monte Carlo program [148]: both the Sudakov approximation and the full one loop corrections have been implemented in order to provide a tool for the fast evaluation of the approximated $\mathcal{O}(\alpha)$ corrections that also allows to asses the validity of the approximated results [149].

Although the Sudakov corrections are universal, this is not the case for the full NLO electroweak corrections that are supposed to work also for energy scales well below the Sudakov regime. Indeed, the non-logarithmic parts of the corrections are strictly process dependent. Despite the fact that $\mathcal{O}(\alpha)$ corrections are not universal, what could be cast in a processindependent form is the way these corrections are computed, namely the algorithms used to compute virtual loop diagrams, to perform the subtraction of IR singularities, to include ultraviolet renormalization and to compute real corrections. This is the first mandatory step in the direction of the automation of NLO EW calculations.

The development of process-independent algorithms to compute radiative corrections was the starting point also for the automation of QCD NLO corrections. Even if in principle the strategies developed for one loop QCD calculations can be extended to cover also the case of EW corrections, this generalization is not trivial because of several specific aspects of electroweak interactions, such as for example the chiral structure of weak interactions (which requires a consistent treatment of $\gamma^{5}$ matrices in loop diagrams), the treatment of unstable particles (that leads to the introduction of complex masses and -as result- of complex couplings), the highly non trivial interplay between QED and QCD-like singularities or the presence of very different mass scales in loop diagrams. On the real emission side the presence of several mass scales and unstable particles typically leads to a phase space with a more involved structure containing Breit-Wigner peaks compared for instance to massless QCD.

Quite recently the issue of automation of one loop electroweak corrections has been addressed by several collaborations and tools like MADGrAPH5_AMC@NLO, OpenLoops and RECOLA have been developed and applied to perform phenomenological studies on the impact of the NLO EW corrections to the processes $p p \rightarrow t \bar{t}+\{H, Z, W\}, p p \rightarrow W+n$ jets $(n=1,2,3)$ and $p p \rightarrow Z(l \bar{l})+2$ jets, respectively.

In Ref. [138] the factorized NLO QCD+EW corrections to the processes $p p \rightarrow W^{+}+1,2$ and 3 jets have been computed using OPENLOOPS interfaced with MUNICH and SHERPA. 
OpenLoops computes LO and NLO amplitudes by means of four-dimensional recursion relations applied at the level of Feynman diagrams. In particular, one loop diagrams are written in terms of tensor integrals multiplied by coefficients that are determined numerically. The tensor integrals are then reduced to scalar integrals by means of the COLLIER library [150] which implements the algorithms of Refs. [151, 152]. Rational terms and renormalization counterterms are included as additional tree-level Feynman rules. MUNICH and SHERPA compute real QCD/QED corrections and perform the subtraction of infrared singularities within the dipole subtraction scheme [153, 154].

In Ref. [137] the one loop corrections of absolute order $\alpha_{S}^{2} \alpha^{3}$ to $Z+2$ jets have been computed using the code RECOLA. Also in RECOLA the computation of LO and NLO amplitudes is based on recursion relations which however act on off-shell currents (that correspond to sum of Feynman sub- diagrams), leading to a different treatment of color with respect to OpenLoops. As in OpenLoops, the algorithm constructs numerically in four dimensions the coefficients of the tensor integrals that are then reduced using the COLLIER library. Rational terms (as well as UV renormalization counterterms) are introduced at the level of LO amplitude generation, while IR singularities are canceled in the dipole scheme.

One loop electroweak and QCD corrections to the processes $p p \rightarrow t \bar{t}+H, p p \rightarrow t \bar{t}+$ $W$ and $p p \rightarrow t \bar{t}+Z$ have been computed in the MADGRAPH5_AMC@NLO framework in Refs. [135, 139]. IR singularities are subtracted in the FKS [155, 156] scheme implemented in MADFKS [157], while one loop amplitudes are generated by MADLOOP which provides an independent implementation of the OpenLoops algorithm (where one loop integrals are computed either via tensor integral reduction or by means of the OPP method).

As a final remark, it is worth noting that the automation of one loop EW corrections is still in its early stages, but rapidly evolving.

\section{VIRTUAL CORRECTIONS AND SCHEME DEPENDENCE}

In one formula, the basic ingredients of an NLO computation can be sketched as

$$
\sigma_{n}^{\mathrm{NLO}}=\sigma_{n}^{\text {Virt. }}+\sigma_{n}^{\mathrm{CT}}+\sigma_{n}^{\text {Real, sub }}+\int \mathrm{d} \Phi^{\mathrm{rad}}\left(\sigma_{n+1}^{\text {Real }}-\sigma_{n+1}^{\mathrm{sub}}\right),
$$

where $n$ is the number of external legs of the LO process under consideration. In Eq. (1),$\sigma_{n}^{\text {Virt. }}$ is the contribution of the diagrams with one virtual loop: these diagrams can be ultraviolet 
(UV) divergent when the number of propagators involved in the loop integral is smaller than four, so that a regularization procedure is needed in order to keep track of the UV singularities that will be removed by means of renormalization (in additive renormalization prescriptions, this is achieved by adding the appropriate counterterms $-\sigma_{n}^{\mathrm{CT}}$ in Eq. (1)- to the virtual corrections). Besides the UV singularities, in massless gauge theories such as QED or QCD, $\sigma_{n}^{\text {Virt. }}$ can be also infrared (IR) divergent: the IR singularities of the virtual one loop corrections are cancelled by the corresponding IR singularities that appear in the computation of real NLO corrections. In Eq. (1) we assume that the integral over the real phase space has been regularized by means of a subtraction procedure [158], such as the Catani-Seymour [153, 154] or the FKS subtraction [155, 156], and $\sigma_{n}^{\text {Real, sub }}$ represents the integrated contribution of the singular part of the real NLO corrections. In the context of NLO corrections to hadronic processes, additional singularities arise due to the collinear splitting of massless initial state partons: as these initial state singularities are universal, they can be absorbed in the parton distribution functions (PDFs) and have to be consistently subtracted form the one loop calculation by adding to Eq. (1) the corresponding collinear counterterms.

The commonly used regularization prescription for UV singularities is dimensional regularization (DR) [159]. The basic idea of DR is to promote the number of space-time dimensions from 4 to $D=4-2 \epsilon_{\mathrm{UV}}<4$, so that the loop diagrams read

$$
\frac{(2 \pi \mu)^{4-D}}{i \pi^{2}} \int \mathrm{d}^{D} q \frac{\mathcal{N}\left(q,\left\{p_{i}, \psi\left(p_{i}\right)\right\}\right)}{\prod_{l=0}^{N}\left[\left(q-r_{l}\right)^{2}-m_{l}^{2}\right]},
$$

where $q$ is the loop momentum, $\left\{p_{i}, \psi\left(p_{i}\right)\right\}$ schematically denote the external momenta (considered as incoming) and their spinors or polarization vectors, $r_{l}=\sum_{i=1, l} p_{i}, m_{l}$ is the mass of the particle in $l$-th loop propagator and $\mu$ is a mass parameter introduced in order to keep the dimension of the integral fixed for varying $D$. In DR singularities appear as $1 / \epsilon_{\mathrm{UV}}$ poles in the limit $D \rightarrow 4$. In Eq. (2) the full Lorentz and Dirac structure of the loop diagram is included in the numerator $\mathcal{N}$. In QCD calculations, DR is the standard regularization prescription also for IR singularities (even if $D=4-2 \epsilon_{\mathrm{IR}}>4$ and $\epsilon_{\mathrm{IR}}=-\epsilon_{\mathrm{UV}}$ ). In the context of NLO electroweak computations, the traditional IR regularization scheme is mass regularization (i.e. IR singularities are regularized by giving an unphysical mass to the photon and the massless fermions), however DR is becoming the new standard procedure in order to treat the IR limit of QED and QCD on the same footing (this is of particular importance not only for the computation of mixed QCD and electroweak corrections, but also for the extension to 
electroweak computations of NLO+parton shower matching algorithms originally developed for QCD calculations [33, 36, 59, 61, 160]).

In the original formulation of DR in Ref. [159], not only the loop momentum but also all the other momenta become $D$-dimensional together with the Lorentz covariants (such as $g^{\mu \nu}$ and $\gamma^{\mu}$ ), which become formal objects obeying specific algebraic relations. This kind of continuation to $D$ dimension is consistent and well defined for non-chiral theories, while problems arise in the treatment of the $\gamma^{5}$ matrix and the $\varepsilon_{\mu \nu \rho \sigma}$ antisymmetric tensor. More precisely, already in Ref. [159] has been pointed out that the main properties of the $\gamma^{5}$ matrix in four dimensions (i.e. $\left\{\gamma^{5}, \gamma^{\mu}\right\}=0, \operatorname{Tr}\left(\gamma^{5} \gamma^{\mu} \gamma^{\nu} \gamma^{\rho} \gamma^{\sigma}\right)=4 i \varepsilon_{\mu \nu \rho \sigma}$ together with the trace cyclicity), in $D$ dimension could lead to algebraic inconsistencies in presence of anomalies. In the literature, several different recipes to handle the $\gamma^{5}$ matrix in the context of DR have been proposed:

- 't Hooft $\gamma^{5}$ prescription. This $\gamma^{5}$ prescription has been proposed by 't Hooft and Veltman [159], Akyeampong and Delbourgo [161, 162] and systematized by Breitenlohner and Maison [163]. According to Ref. [159]:

$$
\begin{gathered}
\gamma^{5}=i \gamma^{0} \gamma^{1} \gamma^{2} \gamma^{3}=\frac{i}{4 !} \varepsilon_{\mu \nu \rho \sigma} \hat{\gamma}^{\mu} \hat{\gamma}^{\nu} \hat{\gamma}^{\rho} \hat{\gamma}^{\sigma}, \\
\left\{\gamma^{5}, \gamma^{\mu}\right\} \neq 0, \quad\left\{\gamma^{5}, \hat{\gamma}^{\mu}\right\}=0, \quad\left[\gamma^{5}, \tilde{\gamma}^{\mu}\right]=0 .
\end{gathered}
$$

In equation (4) we introduced the notation $\gamma^{\mu}=\hat{\gamma}^{\mu}+\tilde{\gamma}^{\mu}$, where $\hat{\gamma}^{\mu}$ and $\tilde{\gamma}^{\mu}$ represent 4 and $D-4$ dimensional objects, respectively. Even if algebraically consistent, the 't Hooft $\gamma^{5}$ prescription leads to spurious anomalies that should be removed by introducing appropriate finite counterterms.

- KKS prescription. The scheme proposed in Refs. [164 166] preserves the anticommutation relationship $\left\{\gamma^{5}, \gamma^{\mu}\right\}=0$ and prevented cyclic property in Dirac traces to avoid algebraic inconsistency. A projection on four-dimensional subspaces is needed in the redefinition of trace operation:

$$
\operatorname{Tr}\left(\gamma^{5} \gamma^{\mu} \gamma^{\nu} \gamma^{\rho} \gamma^{\sigma}\right)=\operatorname{Tr}\left(\mathcal{P}\left(\gamma^{5}\right) \gamma^{\mu} \gamma^{\nu} \gamma^{\rho} \gamma^{\sigma}\right), \quad \mathcal{P}\left(\gamma^{5}\right)=\frac{i}{4 !} \varepsilon_{\mu \nu \rho \sigma} \hat{\gamma}^{\mu} \hat{\gamma}^{\nu} \hat{\gamma}^{\rho} \hat{\gamma}^{\sigma}
$$

Since no cyclic permutation of Dirac matrix inside traces is allowed, all the traces must be read starting from the same vertex (reading point prescription). 
- Naive DR. In this prescription $\left\{\gamma^{5}, \gamma^{\mu}\right\}=0, \operatorname{Tr}\left(\gamma^{5} \gamma^{\mu} \gamma^{\nu} \gamma^{\rho} \gamma^{\sigma}\right)=4 i \varepsilon_{\mu \nu \rho \sigma}$ and cyclic permutation of gamma matrices inside traces are allowed (see for instance Ref. [167]). Even though these properties can be incompatible, the resulting ambiguities are expected to cancel in non-anomalous theories [168 170]. We will take the formulae for the electroweak counterterms from Ref. [171], where the naive prescription has been used: accordingly we will use this prescription in the examples of subsection IID for our CDR (conventional DR) results.

These three different prescriptions for the treatment of $\gamma^{5}$ are three possible examples of different realizations of DR: all of them share the same definition of the denominator of the loop diagram in Eq. (2), while they differ in the treatment of the numerator of Eq. (2). Different choices about the numerator algebra in Eq. (2) concerning the dimension of the momenta, the Dirac matrices or the spinors and polarization vectors, define different DR schemes, such as for example the conventional DR scheme (CDR) [159, 172 174] (or some variant thereof), the 't Hooft Veltman (HV) scheme [159] or the Four Dimensional Helicity scheme (FDH) [175, 176]. Dimensional Reduction (DRED) [177 180] can be regarded as a DR scheme in which all the objects appearing in the numerator of Eq. (2) are four dimensional (except, of course, the loop momentum): this is the regularization scheme used by GoSAM for the computation of one loop diagrams and it is particularly appealing because it allows to work out the numerator of Eq. (2) using four dimensional relations plus some trivial algebra to treat the loop momentum components in the extra dimensions without introducing special Feynman rules (for the finite integrals involved see for example Ref. [14]).

Different DR schemes lead to different results for the computation of one loop diagrams. The differences consist of $\epsilon$-dependent terms, that vanish in the $D \rightarrow 4$ limit unless they multiply the $1 / \epsilon$ poles, giving rise to finite terms (usually called rational terms in the literature). The main purpose of the present paper is to show how the automation of NLO electroweak corrections can be achieved starting from existing tools, such as GoSAM (for the computation of one loop matrix elements) and MADDipole (for the IR subtraction). If we recall Eq. (1), one

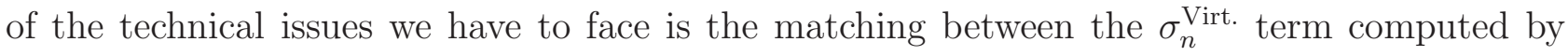
GOSAM in DRED and the contributions coming from renormalization and from the integrated dipoles ( $\sigma_{n}^{\mathrm{CT}}$ and $\sigma_{n}^{\text {Real, sub }}$, respectively) that are usually computed in the CDR scheme in the literature. Although the different building blocks of an NLO calculation are regularization 
scheme dependent, as pointed out in Ref. [181] unitarity implies that the regularization scheme dependence vanishes in the sum of $\sigma_{n}^{\text {Virt. }}, \sigma_{n}^{\mathrm{CT}}$ and $\sigma_{n}^{\text {Real, sub }}$, once these three terms have been computed within the same regularization scheme. For the counterterms part, in particular, we implemented the formulae of Ref. [171] for the on-shell renormalization scheme and derived the conversion factors from CDR to DRED. The infrared subtraction procedure implemented in MADDIPOLE is based on the Catani-Seymour dipole formalism: the CDR to DRED conversion factors for the integrated dipoles have been taken from Ref. [181] (with a straightforward generalization to the case of QED radiation). In subsection $I \mathrm{~A}$ we give a short overview about the electroweak renormalization at one loop in the on-shell scheme, we also explain our treatment of massless fermions and list the relevant regularization scheme conversion factors for the electroweak counterterms. The subtraction of IR singularities is described in subsection while in subsection $\amalg \mathrm{C}$ we address the issue of the regularization scheme dependence of the infrared subtraction procedure. As examples on how the things work, in subsection IID we show the CDR and DRED results for the NLO electroweak corrections to three simple processes: $Z \rightarrow \nu \bar{\nu}, W^{+} \rightarrow u \bar{d}$ and $u \bar{u} \rightarrow c \bar{c}$. In particular, with the examples we show the regularization scheme independence of the sum of virtual one loop corrections, renormalization counterterms and integrated dipoles when these three contributions are consistently computed in the CDR or DRED scheme.

\section{A. Electroweak renormalization and counterterms}

We use the on-shell scheme [182 191] for the electroweak renormalization at one loop. For the sake of simplicity, we employ the notation of Ref. [171] and refer to Appendix B of [171] for the explicit expressions of the self energies involved in the formulas for the electroweak counterterms. Each free parameter of the Standard Model Lagrangian is considered as a bare parameter and it is split into its renormalized version and the corresponding counterterm, namely:

$$
\begin{aligned}
e_{0} & =e+\delta e=\left(1+\delta Z_{e}\right) e, \quad m_{f, 0}=m_{f}+\delta m_{f} \\
M_{W, 0}^{2} & =M_{W}^{2}+\delta M_{W}^{2}, \quad M_{Z, 0}^{2}=M_{Z}^{2}+\delta M_{Z}^{2}, \quad M_{H, 0}^{2}=M_{H}^{2}+\delta M_{H}^{2},
\end{aligned}
$$


(where $f$ stands for a generic fermion field), then for each external field we introduce the corresponding wave function renormalization counterterm:

$$
\begin{aligned}
W_{0} & =\left(1+\frac{1}{2} \delta Z_{W}\right) W, \quad H_{0}=\left(1+\frac{1}{2} \delta Z_{H}\right) H, \\
f_{0}^{L} & =\left(1+\frac{1}{2} \delta Z_{f}^{L}\right) f^{L}, \quad f_{0}^{R}=\left(1+\frac{1}{2} \delta Z_{f}^{R}\right) f^{R}, \\
Z_{0} & =\left(1+\frac{1}{2} \delta Z_{Z Z}\right) Z+\frac{1}{2} \delta Z_{Z A} A, \\
A_{0} & =\left(1+\frac{1}{2} \delta Z_{A A}\right) A+\frac{1}{2} \delta Z_{A Z} Z .
\end{aligned}
$$

This way the Lagrangian can be split into the basic Lagrangian and the counterterm Lagrangian $\mathcal{L}_{0}=\mathcal{L}+\delta \mathcal{L}$, where $\delta \mathcal{L}$ provides the Feynman rules for the counterterms.

In the on-shell scheme the form of the renormalization counterterms is fixed by the following requirements:

- the renormalized masses are the real parts of the poles of the propagators at one loop;

- the residues of the renormalized propagators are equal to one;

- the renormalized electric charge is obtained from the eer vertex in the Thomson limit;

- the renormalized Higgs tadpole is set to zero.

With the above conditions the one loop EW counterterms for fermions, gauge bosons and EW couplings read:

$$
\begin{aligned}
\delta m_{f} & =\frac{m_{f}}{2} \tilde{\operatorname{Re}}\left(\Sigma_{i i}^{f, L}\left(m_{f}^{2}\right)+\Sigma_{i i}^{f, R}\left(m_{f}^{2}\right)+2 \Sigma_{i i}^{f, S}\left(m_{f}^{2}\right)\right) \\
\delta Z_{i i}^{f, L} & =-\tilde{\operatorname{Re}} \Sigma_{i i}^{f, L}\left(m_{f}^{2}\right)-\left.m_{f}^{2} \frac{\partial}{\partial p^{2}}\left(\tilde{\operatorname{Re}} \Sigma_{i i}^{f, L}\left(p^{2}\right)+\tilde{\operatorname{Re}} \Sigma_{i i}^{f, R}\left(p^{2}\right)+2 \tilde{\operatorname{Re}} \Sigma_{i i}^{f, S}\left(p^{2}\right)\right)\right|_{p^{2}=m_{f}^{2}} \\
\delta Z_{i i}^{f, R} & =-\tilde{\operatorname{Re}} \Sigma_{i i}^{f, R}\left(m_{f}^{2}\right)-\left.m_{f}^{2} \frac{\partial}{\partial p^{2}}\left(\tilde{\operatorname{Re}} \Sigma_{i i}^{f, L}\left(p^{2}\right)+\tilde{\operatorname{Re}} \Sigma_{i i}^{f, R}\left(p^{2}\right)+2 \tilde{\operatorname{Re}} \Sigma_{i i}^{f, S}\left(p^{2}\right)\right)\right|_{p^{2}=m_{f}^{2}}
\end{aligned}
$$




$$
\begin{gathered}
\delta M_{W}^{2}=\tilde{\operatorname{Re}} \Sigma_{T}^{W}\left(M_{W}^{2}\right), \quad \delta Z_{W}=-\left.\frac{\partial}{\partial k^{2}} \tilde{\operatorname{Re}} \Sigma_{T}^{W}\left(k^{2}\right)\right|_{k^{2}=M_{W}^{2}}, \\
\delta M_{Z}^{2}=\tilde{\operatorname{Re}} \Sigma_{T}^{Z Z}\left(M_{Z}^{2}\right), \quad \delta Z_{Z Z}=-\left.\frac{\partial}{\partial k^{2}} \tilde{\operatorname{Re}} \Sigma_{T}^{Z Z}\left(k^{2}\right)\right|_{k^{2}=M_{Z}^{2}}, \\
\delta Z_{A A}=-\left.\frac{\partial}{\partial k^{2}} \Sigma_{T}^{A A}\left(k^{2}\right)\right|_{k^{2}=0}, \\
\delta Z_{A Z}=-2 \tilde{\operatorname{Re}} \frac{\Sigma_{T}^{A Z}\left(M_{Z}^{2}\right)}{M_{Z}^{2}}, \quad \delta Z_{Z A}=2 \frac{\Sigma_{T}^{A Z}(0)}{M_{Z}^{2}}, \\
\delta M_{H}^{2}=\tilde{\operatorname{Re}} \Sigma^{H}\left(M_{H}^{2}\right), \quad \delta Z_{H}=-\left.\frac{\partial}{\partial k^{2}} \tilde{\operatorname{Re}} \Sigma^{H}\left(k^{2}\right)\right|_{k^{2}=M_{H}^{2}}, \\
\frac{\delta e}{e}=-\frac{1}{2} \delta Z_{A A}-\frac{s_{W}}{c_{W}} \frac{1}{2} \delta Z_{Z A}=\left.\frac{1}{2} \frac{\partial}{\partial k^{2}} \Sigma_{T}^{A A}\left(k^{2}\right)\right|_{k^{2}=0}-\frac{s_{W}}{c_{W}} \frac{\Sigma_{T}^{A Z}(0)}{M_{Z}^{2}} \\
\frac{\delta c_{W}}{c_{W}}=\frac{1}{2}\left(\frac{\delta M_{W}^{2}}{M_{W}^{2}}-\frac{\delta M_{Z}^{2}}{M_{Z}^{2}}\right)=\frac{1}{2} \tilde{\operatorname{Re}}\left(\frac{\Sigma_{T}^{W}\left(M_{W}^{2}\right)}{M_{W}^{2}}-\frac{\Sigma_{T}^{2}}{M_{Z}^{2}}\right) \\
\frac{\delta s_{W}}{s_{W}}=-\frac{c_{W}^{2}}{s_{W}^{2}} \frac{\delta c_{W}}{c_{W}}=-\frac{1}{2} \frac{c_{W}^{2}}{s_{W}^{2}} \tilde{\operatorname{Re}}\left(\frac{\Sigma_{T}^{W}\left(M_{W}^{2}\right)}{M_{W}^{2}}\right) \\
\delta t=-T^{H} \cdot
\end{gathered}
$$

Where the notation Re means that the real part is taken only for the scalar functions contained in the unrenormalized self energies. As in our computation the CKM matrix $V_{\mathrm{CKM}}$ is chosen to be diagonal and no renormalization of $V_{\mathrm{CKM}}$ is needed, we do not discuss here the renormalization conditions for the quark mixing matrix.

In the following we list the conversion rules from the CDR to the DRED regularization schemes. For electroweak couplings and gauge bosons the conversion rules read:

$$
\begin{aligned}
\delta Z_{e}^{\mathrm{DRED}} & =\delta Z_{e}^{\mathrm{CDR}}+\frac{\alpha}{4 \pi} \frac{1}{3}, \quad \delta Z_{Z A}^{\mathrm{DRED}}=\delta Z_{Z A}^{\mathrm{CDR}}, \quad \delta Z_{A Z}^{\mathrm{DRED}}=\delta Z_{A Z}^{\mathrm{CDR}}+\frac{\alpha}{4 \pi} \frac{4}{3} \frac{c_{W}}{s_{W}} \\
\delta Z_{A A}^{\mathrm{DRED}} & =\delta Z_{A A}^{\mathrm{CDR}}-\frac{\alpha}{4 \pi} \frac{2}{3}, \quad \delta Z_{Z Z}^{\mathrm{DRED}}=\delta Z_{Z Z}^{\mathrm{CDR}}-\frac{\alpha}{4 \pi} \frac{2}{3} \frac{c_{W}^{2}}{s_{W}^{2}}, \quad \delta Z_{W}^{\mathrm{DRED}}=\delta Z_{W}^{\mathrm{CDR}}-\frac{\alpha}{4 \pi} \frac{2}{3 s_{W}^{2}} \\
\delta M_{W}^{2 \mathrm{DRED}} & =\delta M_{W}^{2 \mathrm{CDR}}+\frac{\alpha}{4 \pi} \frac{2}{3} \frac{M_{W}^{2}}{s_{W}^{2}}, \quad \delta M_{Z}^{2 \mathrm{DRED}}=\delta M_{Z}^{2 \mathrm{CDR}}+\frac{\alpha}{4 \pi} \frac{2}{3} \frac{M_{Z}^{2} c_{W}^{2}}{s_{W}^{2}} \\
\delta Z_{H}^{\mathrm{DRED}} & =\delta Z_{H}^{\mathrm{CDR}}, \quad \delta M_{H}^{2 \mathrm{DRED}}=\delta M_{H}^{2 \mathrm{CDR}}+\frac{\alpha}{4 \pi} \frac{3\left(2 c_{W}^{2} M_{W}^{2}+M_{Z}^{2}\right)}{2 c_{W}^{2} s_{W}^{2}} .
\end{aligned}
$$


The CDR to DRED conversion rules for up-type quarks, down-type quarks and leptons, respectively, are:

$$
\begin{aligned}
\delta m_{u}^{\mathrm{DRED}} & =\delta m_{u}^{\mathrm{CDR}}+\frac{\alpha}{4 \pi} m_{u} \frac{\left(3-4 s_{W}^{2}\right)^{2}+2 c_{W}^{2}\left(9+8 s_{W}^{2}\right)}{72 c_{W}^{2} s_{W}^{2}}, \\
\delta Z_{u}^{L \mathrm{DRED}} & =\delta Z_{u}^{L \mathrm{CDR}}-\frac{\alpha}{4 \pi} \frac{\left(3-4 s_{W}^{2}\right)^{2}+2 c_{W}^{2}\left(9+8 s_{W}^{2}\right)}{36 c_{W}^{2} s_{W}^{2}} \\
\delta Z_{u}^{R \mathrm{DRED}} & =\delta Z_{u}^{R \mathrm{CDR}}-\frac{\alpha}{4 \pi} \frac{4}{9 c_{W}^{2}} ; \\
\delta m_{d}^{\mathrm{DRED}} & =\delta m_{d}^{\mathrm{CDR}}+\frac{\alpha}{4 \pi} m_{d} \frac{9+4 s_{W}^{4}+2 c_{W}^{2}\left(9+2 s_{W}^{2}\right)}{72 c_{W}^{2} s_{W}^{2}}, \\
\delta Z_{d}^{L \mathrm{DRED}} & =\delta Z_{d}^{L \mathrm{CDR}}-\frac{\alpha}{4 \pi} \frac{\left(3-2 s_{W}^{2}\right)^{2}+2 c_{W}^{2}\left(9+2 s_{W}^{2}\right)}{36 c_{W}^{2} s_{W}^{2}}, \\
\delta Z_{d}^{R \mathrm{DRED}} & =\delta Z_{d}^{R \mathrm{CDR}}-\frac{\alpha}{4 \pi} \frac{1}{9 c_{W}^{2}} ; \\
\delta m_{e}^{\mathrm{DRED}} & =\delta m_{e}^{\mathrm{CDR}}+\frac{\alpha}{4 \pi} m_{e} \frac{1+4 s_{W}^{2}\left(s_{W}^{2}-2\right)+c_{W}^{2}\left(2+4 s_{W}^{2}\right)}{8 c_{W}^{2} s_{W}^{2}} \\
\delta Z_{e}^{L \mathrm{DRED}} & =\delta Z_{e}^{L \mathrm{CDR}}-\frac{\alpha}{4 \pi} \frac{1+2 c_{W}^{2}}{4 c_{W}^{2} s_{W}^{2}}, \\
\delta Z_{e}^{R \mathrm{DRED}} & =\delta Z_{e}^{R \mathrm{CDR}}-\frac{\alpha}{4 \pi} \frac{1}{c_{W}^{2}}, \\
\delta Z_{\nu}^{L \mathrm{DRED}} & =\delta Z_{\nu}^{L \mathrm{CDR}}-\frac{\alpha}{4 \pi} \frac{1+2 c_{W}^{2}}{4 c_{W}^{2} s_{W}^{2}} .
\end{aligned}
$$

As in our numerical examples we deal with external massless quarks, following for example Ref. [59], we adopt a slightly modified version of the on-shell scheme where the light quark masses are set to zero in the corresponding external wave function renormalization counterterms (and mass counterterms for light quarks vanish). In this approach eqs. (12)-(13) for massless up and down-type quarks in particular become:

$$
\begin{aligned}
\delta Z_{u}^{L \mathrm{DRED}} & =\delta Z_{u}^{L \mathrm{CDR}}-\frac{\alpha}{4 \pi} \frac{\left(3-4 s_{W}^{2}\right)^{2}+18 c_{W}^{2}}{36 c_{W}^{2} s_{W}^{2}}, \\
\delta Z_{u}^{R \mathrm{DRED}} & =\delta Z_{u}^{R \mathrm{CDR}}-\frac{\alpha}{4 \pi} \frac{4 s_{W}^{2}}{9 c_{W}^{2}} ; \\
\delta Z_{d}^{L \mathrm{DRED}} & =\delta Z_{d}^{L \mathrm{CDR}}-\frac{\alpha}{4 \pi} \frac{\left(3-2 s_{W}^{2}\right)^{2}+18 c_{W}^{2}}{36 c_{W}^{2} s_{W}^{2}}, \\
\delta Z_{d}^{R \mathrm{DRED}} & =\delta Z_{d}^{R \mathrm{CDR}}-\frac{\alpha}{4 \pi} \frac{s_{W}^{2}}{9 c_{W}^{2}} .
\end{aligned}
$$


Large logarithms of the fermion masses appear in the counterterms $\delta Z_{e}$ and $\delta Z_{A A}$. They are basically related to the running of $\alpha$ from the Thomson limit to the electroweak scale. These terms are singular in the limit $m_{q} \rightarrow 0$, however they can be systematically cancelled by following the recommendation of the $E W$ dictionary of Ref. [62], namely by choosing $\alpha(0)$ as coupling constant for the LO vertices involving external photons and $\alpha\left(M_{Z}^{2}\right)$ or $\alpha_{G_{\mu}}$ for the remaining LO vertices. In the former case the logarithms of the light fermion masses cancel in the combination $\delta Z_{e}-\frac{1}{2} \delta Z_{A A}$, while in the latter they vanish as a result of the finite shifts $\left.\left.\delta Z^{e}\right|_{\alpha\left(M_{Z}^{2}\right)} \rightarrow \delta Z^{e}\right|_{\alpha(0)}-\frac{1}{2} \Delta \alpha\left(M_{Z}^{2}\right),\left.\left.\delta Z^{e}\right|_{G_{\mu}} \rightarrow \delta Z^{e}\right|_{\alpha(0)}-\frac{1}{2} \Delta r$ [49, 119, 171]. The remaining dependence on the light quark masses in the counterterms vanishes in the $m_{q} \rightarrow 0$ limit. Indeed, in this approach light quark masses can be safely neglected from the very beginning.

We conclude this section with a short remark on unstable particles. In general the description of resonances in perturbation theory requires a Dyson summation of the self energy insertions. This leads to a mixing of perturbative orders that could break gauge invariance. In the context on one loop electroweak corrections unstable particles are usually treated within the so-called complex mass scheme (CMS) 192 194]. In the CMS unstable particles masses are promoted to complex numbers through the replacement

$$
m_{V}^{2} \rightarrow \mu_{V}^{2}=m_{V}^{2}-i m_{V} \Gamma_{V}
$$

(where $\Gamma_{V}$ is the decay width of the unstable particle $V$ ) and the complex Weinberg angle is then defined via

$$
\cos ^{2} \theta_{W}=\frac{\mu_{W}^{2}}{\mu_{Z}^{2}} .
$$

Within the CMS renormalization can be performed in a modified version of the on shell scheme. The renormalized masses of the unstable particles are defined as the poles of the corresponding propagators in the complex plane. The resulting expressions for the counterterms are basically the same as in eqs. (8,10) with the important difference that all the self energies involved become complex (via their dependence on the complex couplings and masses) and no real part is taken. In the CMS also the momentum flowing in the self energies is a complex variable. However, it is possible to realize a minimal version of the on shell renormalization in the CMS by expanding the self energies about the real arguments in such a way that one loop accuracy is retained. This in particular requires the introduction of an additional renormalization factor for the $W$ (and also the top) self energy in order to deal with the branch cut at $k^{2}=\mu_{W(t)}^{2}$ 
arising from the photonic loop diagrams. The resulting counterterms are still complex, but depend on real momenta.

\section{B. Electroweak insertion operator}

In equation (1) we briefly sketched the basic building blocks of an NLO calculation. We pointed out that virtual corrections $\sigma_{n}^{\text {Virt. }}$ and real contributions $\sigma_{n+1}^{\text {Real }}$ are separately infrared divergent and the IR singularities only cancel in the sum of the two terms (as far as infrared safe observables are considered and the initial state collinear singularities are properly absorbed in the PDFs in the case of hadronic processes). In Eq. (1) we also introduced the infrared subtraction term $\sigma_{n+1}^{\text {sub }}$ and its integrated counterpart $\sigma_{n}^{\text {Real, sub }} \cdot \sigma_{n+1}^{\text {sub }}$ is a function of the $n+1$ kinematics that behaves as the matrix element for the real corrections in the soft/collinear limit: this way $\sigma_{n+1}^{\text {Real }}-\sigma_{n+1}^{\text {sub }}$ in Eq. (11) is IR finite and can be integrated numerically. $\sigma_{n+1}^{\text {sub }}$ should also have a simple expression, so that the IR subtraction term can be integrated analytically

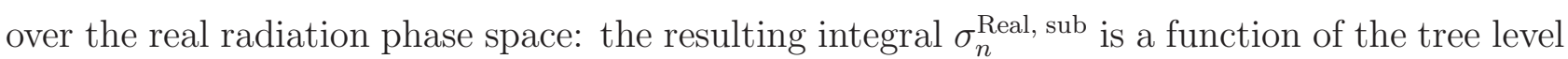
$n$ particle kinematics that has to be added back to the virtual corrections in order to cancel the IR poles of the one loop contributions.

Owing to the universality of the infrared limit of massless gauge theories like QCD it is possible to develop process independent algorithms to build the IR subtraction terms and of course their integrated counterparts (the dipole subtraction or the FKS methods are two examples of such algorithms). The infrared properties of one loop amplitudes are well known and in the context of QCD are usually described by a so called insertion operator [195], that corresponds to the integrated subtraction terms described above. These considerations can be easily translated to electroweak one loop amplitudes and the infrared properties of such amplitudes are then described by an electroweak insertion operator. The electroweak infrared insertion operator is particularly useful as it allows to check the cancellation of double and single pole of the renormalized amplitude for a given phase space point. It can be obtained for instance from the results of [153, 154] by, simply speaking, replacing the color factors by electric charges. More specifically we start by defining the singular pieces as:

$$
\mathcal{V}_{\text {sing }, Q E D}\left(s_{i k}, m_{i}, m_{k}\right)=\frac{\alpha}{2 \pi}\left(\frac{1}{\epsilon^{2}} \mathcal{V}_{\epsilon^{2}}\left(s_{i k}, m_{i}, m_{k}\right)+\frac{1}{\epsilon} \mathcal{V}_{\epsilon}\left(s_{i k}, m_{i}, m_{k}\right)\right) \cdot\left|\mathcal{M}_{\text {Born }}\right|^{2}
$$

The index $i$ denotes the combination of emitter and unresolved particle, the index $k$ denotes 
the spectator. The coefficients of the double and the single pole depend on the masses and are given by

$$
\begin{aligned}
m_{i}>0, m_{k}>0 & \\
& \mathcal{V}_{\epsilon^{2}}\left(s_{i k}, m_{i}, m_{k}\right)=0 \\
\mathcal{V}_{\epsilon}\left(s_{i k}, m_{i}, m_{k}\right)=\frac{\log \rho}{v_{i k}}, \quad v_{i k}=\frac{\sqrt{\lambda\left(s_{i k}, m_{i}^{2}, m_{k}^{2}\right)}}{s_{i k}-m_{i}^{2}-m_{k}^{2}}, \rho=\sqrt{\frac{1-v_{i k}}{1+v_{i} k},} & \lambda(x, y, z)=x^{2}+y^{2}+z^{2}-2 x y-2 y z-2 x z, \\
m_{i}>0, m_{k}=0: & \\
& \mathcal{V}_{\epsilon^{2}}\left(s_{i k}, m_{i}, 0\right)=\frac{1}{2} \\
& \mathcal{V}_{\epsilon}\left(s_{i k}, m_{i}, 0\right)=\frac{1}{2}\left(\frac{5}{2}+\log \frac{m_{i}^{2}}{s_{i k}}\right) \\
m_{i}=0, m_{k}>0: & \mathcal{V}_{\epsilon^{2}}\left(s_{i k}, 0, m_{k}\right)=\frac{1}{2} \\
& \mathcal{V}_{\epsilon}\left(s_{i k}, 0, m_{k}\right)=\frac{1}{2}\left(\frac{5}{2}+\log \frac{m_{k}^{2}}{s_{i k}}\right) \\
m_{i}=0, m_{k}=0: & \mathcal{V}_{\epsilon^{2}}\left(s_{i k}, 0,0\right)=1 \\
& \mathcal{V}_{\epsilon}\left(s_{i k}, 0,0\right)=\frac{3}{2} .
\end{aligned}
$$

The insertion operator depends on the fact whether emitter/spectator are in the initial or final state. In particular, we have:

initial-initial:

$$
\begin{aligned}
& \mathbf{I}_{\epsilon^{2}}\left(s_{i k}, m_{i}, m_{k}\right)=\frac{n_{c} \sigma_{i} q_{i} \sigma_{k} q_{k}}{\epsilon^{2}} \mathcal{V}_{\epsilon^{2}}\left(s_{i k}, m_{i}, m_{k}\right) \cdot\left|\mathcal{M}_{\text {Born }}\right|^{2} \\
& \mathbf{I}_{\epsilon}\left(s_{i k}, m_{i}, m_{k}, \mu^{2}\right)=\frac{n_{c} \sigma_{i} q_{i} \sigma_{k} q_{k}}{\epsilon} \mathcal{V}_{\epsilon}\left(s_{i k}, m_{i}, m_{k}\right)\left(\frac{3}{2}+\log \frac{\mu^{2}}{s_{i k}}\right) \cdot\left|\mathcal{M}_{\text {Born }}\right|^{2}
\end{aligned}
$$

initial-final / final-initial / final-final :

$$
\begin{aligned}
& \mathbf{I}_{\epsilon^{2}}\left(s_{i k}, m_{i}, m_{k}\right)=\frac{n_{c} \sigma_{i} q_{i} \sigma_{k} q_{k}}{\epsilon^{2}} \mathcal{V}_{\epsilon^{2}}\left(s_{i k}, m_{i}, m_{k}\right) \cdot\left|\mathcal{M}_{\text {Born }}\right|^{2} \\
& \mathbf{I}_{\epsilon}\left(s_{i k}, m_{i}, m_{k}, \mu^{2}\right)=\frac{n_{c} \sigma_{i} q_{i} \sigma_{k} q_{k}}{\epsilon}\left(\mathcal{V}_{\epsilon}\left(s_{i k}, m_{i}, m_{k}\right)+\log \frac{\mu^{2}}{s_{i k}} \mathcal{V}_{\epsilon^{2}}\left(s_{i k}, m_{i}, m_{k}\right)\right) \cdot\left|\mathcal{M}_{\text {Born }}\right|^{2} .
\end{aligned}
$$


The factors $q_{i}, q_{k}$ denote the fractional electric charges of emitter and spectator and $n_{c}$ is the number of colors that can occur in the splitting (i.e. either one or three). In addition, we follow Ref.[196] and introduce the sign factors $\sigma_{i, k}$ which are defined to be +1 for incoming fermions and outgoing anti-fermions and -1 for incoming anti-fermions and outgoing fermions. This can be extended to the case of a final state $W$-boson and yields a +1 for an external $W^{-}$and a -1 for an external $W^{+}$. The sign factors ensure the conservation of the electric charge which is given by

$$
\sum_{n} \sigma_{n} \cdot q_{n}=0
$$

\section{Subtraction terms and scheme dependence}

Numerical simulations require the use of subtraction terms to render the real emission contribution finite. One of the widely used subtraction methods in the context of electroweak calculation is the dipole formalism [153] which has been adapted to electroweak calculations [196, 197]. In practice the subtraction methods usually also takes care of issues regarding the regularization scheme by adding appropriate additional terms to cancel the scheme dependence in the virtual corrections.

As mentioned above, different regularization schemes lead to differences in the finite contribution for the virtual corrections. At the one-loop level the transition from one scheme to the other can be obtained by a simple shift that is proportional to the Born contribution [181]. Following the derivation of Ref. [181], in the case of photon emission one obtains a conversion term of the form:

$$
\delta_{R S}=-\frac{1}{2} q_{i} \sigma_{i} q_{k} \sigma_{k}
$$

changing from CDR to DRED. Here $i$ denotes the emitter, $k$ denotes the spectator. $q_{i, k}$ are the fractional charges of emitter and spectator respectively, and $\sigma_{i, k}$ are the sign factors as defined in Eq. (22). However there is only a contribution in case of massless emitters. Soft singularities are independent of the scheme and therefore a scheme dependence only arises from collinear splittings. As these are finite in the massive case there is no transition term for massive emitters. Eventually the full transition term is obtained by summing over all possible emitter-spectator pairs. 

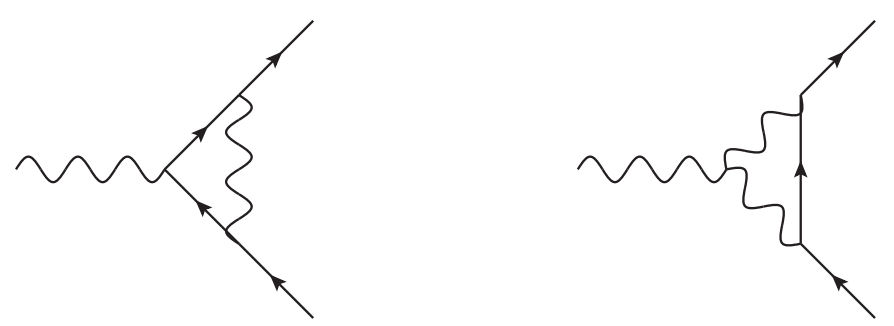

FIG. 1: One loop electroweak corrections to $V \rightarrow f \bar{f}^{\prime}$. The diagram on the left gives rise to the $\mathcal{V}_{a}$ contributions, while the $\mathcal{V}_{b}$ terms come from the diagram on the right.

\section{Examples}

$$
\text { 1. } Z \rightarrow \nu \bar{\nu}
$$

As a first simple example of the applications of the formulas of the previous subsections we consider the decay $Z \rightarrow \nu \bar{\nu}$. At the LO the corresponding matrix element is:

$$
\mathcal{M}_{0}=i e g_{\nu}^{-} \bar{u}\left(p_{1}\right) \gamma^{\mu} \omega_{-} v\left(p_{2}\right) \varepsilon_{\mu}\left(p_{1}+p_{2}\right)
$$

where $g_{f}^{-}=\left(I_{f}-s_{W}^{2} Q_{f}\right) / s_{W} c_{W}, \omega_{ \pm}=\left(1 \pm \gamma^{5}\right) / 2$ and $\varepsilon_{\mu}$ is the polarization vector for the on-shell external $Z$ boson.

The two classes of diagrams contributing to the one loop electroweak corrections to $Z \rightarrow \nu \bar{\nu}$ are shown in Fig. 1. The unrenormalized $\mathcal{O}(\alpha)$ corrections to the $Z$ decay in neutrinos read:

$$
\begin{aligned}
& \mathcal{M}_{\text {Virt. }}=\mathcal{M}_{0} \frac{\alpha}{4 \pi}\left\{\frac{1}{4 s_{W}^{2} c_{W}^{2}} \mathcal{V}_{a}\left(0, M_{Z}^{2}, 0, M_{Z}, 0,0\right)\right. \\
& +\frac{s_{W}^{2}-\frac{1}{2}}{s_{W}^{2}} \mathcal{V}_{a}\left(0, M_{Z}^{2}, 0, M_{W}, 0,0\right) \\
& \left.+\frac{c_{W}^{2}}{s_{W}^{2}} \quad \mathcal{V}_{b}\left(0, M_{Z}^{2}, 0,0, M_{W}, M_{W}\right)\right\}
\end{aligned}
$$

where we used the notation of Ref. [171] for the vertex functions $\mathcal{V}_{a(b)}$. It is worth noting that Eq. (25) does not depend on the regularization scheme. What is scheme dependent is the 
expression of the $\mathcal{V}_{a(b)}$ functions. When performing the calculation in CDR we find:

$$
\begin{aligned}
& \mathcal{V}_{a}^{\mathrm{CDR}}(0, s, 0, m, 0,0)=-2 s C_{0}(0, s, 0, m, 0,0)\left(1+\frac{m^{2}}{s}\right)^{2}-B_{0}(s, 0,0)\left(3+2 \frac{m^{2}}{s}\right) \\
&+2 B_{0}(0, m, 0)\left(2+\frac{m^{2}}{s}\right)-2 \\
& \mathcal{V}_{b}^{\mathrm{CDR}}\left(0, s, 0,0, m_{1}, m_{2}\right)=2\left(m_{1}^{2}+m_{2}^{2}+\frac{m_{1}^{2} m_{2}^{2}}{s}\right) C_{0}\left(0, s, 0,0, m_{1}, m_{2}\right) \\
&-B_{0}\left(s, m_{1}, m_{2}\right)\left(1+\frac{m_{1}^{2}+m_{2}^{2}}{s}\right) \\
&+\left(2+\frac{m_{1}^{2}}{s}\right) B_{0}\left(0,0, m_{1}^{2}\right)+\left(2+\frac{m_{2}^{2}}{s}\right) B_{0}\left(0,0, m_{2}^{2}\right),
\end{aligned}
$$

(where $B_{0}$ and $C_{0}$ are the usual Passarino-Veltman two and three point functions, respectively [198 200]), while the DRED results are related to CDR ones as follows:

$$
\begin{gathered}
\mathcal{V}_{a}^{\mathrm{DRED}}(0, s, 0, m, 0,0)=\mathcal{V}_{a}^{\mathrm{CDR}}(0, s, 0, m, 0,0)+1 \\
\mathcal{V}_{b}^{\mathrm{DRED}}\left(0, s, 0,0, m_{1}, m_{2}\right)=\mathcal{V}_{b}^{\mathrm{CDR}}\left(0, s, 0,0, m_{1}, m_{2}\right)+1 .
\end{gathered}
$$

The $\mathcal{O}(\alpha)$ corrections to $Z \rightarrow \nu \bar{\nu}$ coming from renormalization read:

$$
\mathcal{M}_{\mathrm{CT}}=\mathcal{M}_{0}\left\{\frac{1}{2} \delta Z_{Z}+\delta Z_{\nu}^{L}+\delta Z_{e}+\left(\frac{s_{W}^{2}}{c_{W}^{2}}-1\right) \frac{\delta s_{W}}{s_{W}}\right\} .
$$

Using Eq. (28) and the scheme conversion rules for the counterterms in eqs. (11) and (14), it is easy to show that $\left(\frac{\mathcal{M}_{\text {Virt. }}+\mathcal{M}_{\mathrm{CT}}}{\mathcal{M}_{0}}\right)^{\mathrm{CDR}}=\left(\frac{\mathcal{M}_{\text {Virt. }}+\mathcal{M}_{\mathrm{CT}}}{\mathcal{M}_{0}}\right)^{\mathrm{DRED}}$.

Note that Eq. (29) contains large logarithms of of the light fermion masses, that can be removed by using $\alpha\left(M_{Z}^{2}\right)$ as input parameter in Eq. (24) and performing the corresponding finite renormalization $\left.\left.\delta Z^{e}\right|_{\alpha\left(M_{Z}^{2}\right)} \rightarrow \delta Z^{e}\right|_{\alpha(0)}-\frac{1}{2} \Delta \alpha\left(M_{Z}^{2}\right)$, where

$$
\Delta \alpha\left(Q^{2}\right)=\Pi_{f \in \text { light }}^{A A}(0)-\operatorname{Re}\left(\Pi_{f \in \text { light }}^{A A}\left(Q^{2}\right)\right), \quad \Pi_{f \in \text { light }}^{A A}\left(Q^{2}\right)=\frac{\sum_{f \in \text { light }}^{A A}\left(Q^{2}\right)}{Q^{2}},
$$

and $\Sigma_{f \in \text { light }}^{A A}$ represents the contribution of light fermion loops to the photon self energy (which has the same expression both in DRED and in CDR). Once the singular terms in the $m_{q} \rightarrow 0$ limit have been subtracted, the remaining dependence on the light quark masses can be safely neglected.

$$
\text { 2. } W^{+} \rightarrow u \bar{d}
$$

With the notation of Eq. (24), the matrix element for the process $W^{+} \rightarrow u \bar{d}$ is:

$$
\mathcal{M}_{0}=i \frac{e}{\sqrt{2} s_{W}} \bar{u}\left(p_{1}\right) \gamma^{\mu} \omega_{-} v\left(p_{2}\right) \varepsilon_{\mu}\left(p_{1}+p_{2}\right)
$$


The one loop electroweak corrections to $W^{+} \rightarrow u \bar{d}$ are given by the two classes of diagrams in Fig. 1. At variance with the $Z \rightarrow \nu \bar{\nu}$ case, in this case also photon loops contribute to the corrections. We basically follow the computation of Ref. [171], with the important difference that we use dimensional regularization for the IR singularities coming from photon loops. These IR singularities are cancelled by the QED dipole subtraction terms described in subsection IIB.

The unrenormalized virtual one loop matrix element is:

$$
\begin{aligned}
& \mathcal{M}_{\text {Virt. }}=\mathcal{M}_{0} \frac{\alpha}{4 \pi}\left\{Q_{u} Q_{d} \quad \mathcal{V}_{a}\left(0, M_{W}^{2}, 0,0,0,0\right)\right. \\
& +g_{u}^{-} g_{d}^{-} \quad \mathcal{V}_{a}\left(0, M_{W}^{2}, 0, M_{Z}, 0,0\right) \\
& +Q_{u} \quad \mathcal{V}_{b}\left(0, M_{W}^{2}, 0,0,0, M_{W}\right) \\
& -Q_{d} \quad \mathcal{V}_{b}\left(0, M_{W}^{2}, 0,0, M_{W}, 0\right) \\
& +g_{u}^{-} \frac{c_{W}}{s_{W}} \mathcal{V}_{b}\left(0, M_{W}^{2}, 0,0, M_{Z}, M_{W}\right) \\
& \left.-g_{d}^{-} \frac{c_{W}}{s_{W}} \mathcal{V}_{b}\left(0, M_{W}^{2}, 0,0, M_{W}, M_{Z}\right)\right\} \text {, }
\end{aligned}
$$

while the contribution coming from renormalization can be written as:

$$
\mathcal{M}_{\mathrm{CT}}=\mathcal{M}_{0}\left\{\frac{1}{2} \delta Z_{W}+\frac{1}{2} \delta Z_{u}^{L}+\frac{1}{2} \delta Z_{d}^{L}+\delta Z_{e}-\frac{\delta s_{W}}{s_{W}}\right\}
$$

As in the previous example, eqs. (32+33) are completely general, while the expression of the vertex functions and the counterterms depend on the regularization scheme. If we define $\delta^{\text {Virt. }}$ via the relation:

$$
2 \operatorname{Re}\left[\left(\mathcal{M}_{\text {Virt. }}+\mathcal{M}_{\mathrm{CT}}\right)^{*} \mathcal{M}_{0}\right]=\frac{\alpha}{2 \pi} \delta^{\text {Virt. }}\left|\mathcal{M}_{0}\right|^{2}
$$

from eqs. (28), (15,-16) and (11), we find that the one loop virtual electroweak corrections have the following regularization scheme dependence:

$$
\delta_{\mathrm{DRED}}^{\text {Virt. }}-\delta_{\mathrm{CDR}}^{\text {Virt. }}=\frac{1}{2} Q_{u}^{2}+\frac{1}{2} Q_{d}^{2} .
$$

Also in this case, the scheme dependence of the one loop electroweak corrections is canceled by the corresponding scheme dependence of the QED integrated dipoles that are obtained from Eq. (23) by summing over all emitter and spectator pairs. As a result, the sum of the renormalized one loop virtual corrections and the infrared subtraction terms is regularization scheme independent. As in the previous example, the dependence on the light quark masses in Eq. (33) can be removed by using $\alpha\left(M_{Z}^{2}\right)$ or $\alpha_{G_{\mu}}$ as input parameters. 


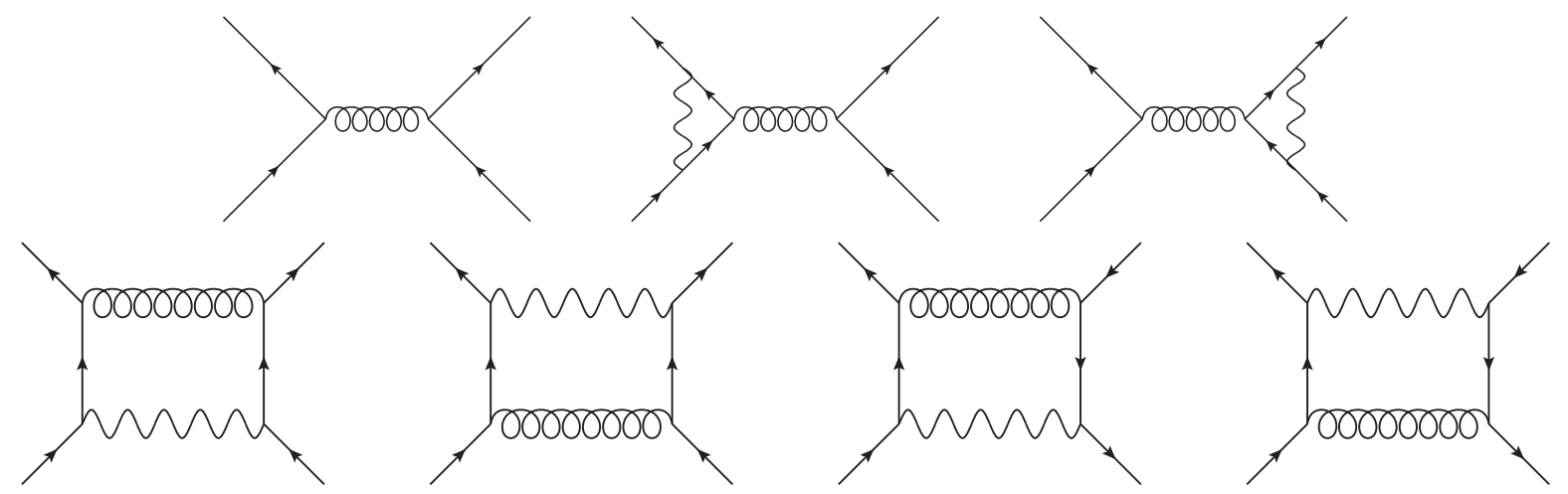

FIG. 2: LO and virtual NLO electroweak diagrams for the process $u \bar{u} \rightarrow c \bar{c}$.

$$
\text { 3. } u \bar{u} \rightarrow c \bar{c}
$$

As a last simple example we consider the one loop electroweak corrections to the $\mathcal{O}\left(\alpha_{S}\right)$ process $u \bar{u} \rightarrow c \bar{c}$. As the $\mathcal{O}(\alpha)$ corrections are helicity-dependent, it is convenient to write the LO matrix element as:

$$
\mathcal{M}_{0}=\frac{i g_{S}^{2} T_{i j}^{a} T_{k l}^{b}}{\left(p_{u}+p_{\bar{u}}\right)^{2}} \sum_{\lambda_{i}, \lambda_{f}= \pm} \bar{u}\left(p_{c}\right) \gamma^{\mu} \omega_{\lambda_{f}} v\left(p_{\bar{c}}\right) \bar{v}\left(p_{\bar{u}}\right) \gamma_{\mu} \omega_{\lambda_{i}} u\left(p_{u}\right)=\sum_{\lambda_{i}, \lambda_{f}= \pm} \mathcal{M}_{0}\left(\lambda_{i}, \lambda_{f}\right) .
$$

Fig. 2 represents the diagrams contributing to the NLO electroweak corrections to the process $u \bar{u} \rightarrow c \bar{c}$. As in the previous example, we regularize dimensionally the QED-like IR singularities coming from the photon loops and also the QCD-like IR singularities associated with the gluon exchange between the fermionic currents in the initial and in the final states. For a given helicity configuration, the virtual electroweak corrections read:

$$
\mathcal{M}_{\text {Virt. }}\left(\lambda_{i}, \lambda_{f}\right)=\mathcal{M}_{0}\left(\lambda_{i}, \lambda_{f}\right) \frac{\alpha}{4 \pi}\left\{\delta^{\operatorname{Vertex}}\left(\lambda_{i}\right)+\delta^{\operatorname{Vertex}}\left(\lambda_{f}\right)+\delta^{\operatorname{Box}}\left(\lambda_{i}, \lambda_{f}\right)\right\},
$$

with

$$
\begin{aligned}
& \delta^{\operatorname{Vertex}}(\lambda)=\left\{\quad\left(g_{u}^{\lambda}\right)^{2} \quad \mathcal{V}_{a}\left(0,\left(p_{u}+p_{\bar{u}}\right)^{2}, 0, M_{Z}, 0,0\right)\right. \\
& +Q_{u}^{2} \quad \mathcal{V}_{a}\left(0,\left(p_{u}+p_{\bar{u}}\right)^{2}, 0,0,0,0\right) \\
& \left.+\delta_{\lambda,-} \frac{1}{4 s_{W}^{2}} \mathcal{V}_{a}\left(0,\left(p_{u}+p_{\bar{u}}\right)^{2}, 0, M_{W}, 0,0\right)\right\} \text {. }
\end{aligned}
$$

The counterterm contribution is:

$$
\mathcal{M}_{\mathrm{CT}}\left(\lambda_{i}, \lambda_{f}\right)=\mathcal{M}_{0}\left(\lambda_{i}, \lambda_{f}\right)\left\{\delta Z_{u}^{\lambda_{i}}+\delta Z_{u}^{\lambda_{f}}\right\}
$$


where $\delta Z_{u}^{+}=\delta Z_{u}^{R}, \delta Z_{u}^{-}=\delta Z_{u}^{L}$ and $\delta Z_{u}^{L(R)}=\delta Z_{c}^{L(R)}$.

It is worth noting that the box contribution in Eq. (37) does not depend on the regularization scheme (CDR/DRED) and does not give rise to rational terms neither of UV type (since it is UV finite) nor of IR type [201]. As a consequence, using eqs. (28) in (38) together with the scheme conversion formulas for the counterterms (15) we can show that for each of the helicity configurations the one loop electroweak corrections depend on the regularization scheme according to:

$$
\delta_{\mathrm{DRED}}^{\text {Virt. }}\left(\lambda_{i}, \lambda_{f}\right)-\delta_{\mathrm{CDR}}^{\text {Virt. }}\left(\lambda_{i}, \lambda_{f}\right)=2 Q_{u}^{2}
$$

where $\delta^{\text {Virt. }}\left(\lambda_{i}, \lambda_{f}\right)$ corresponds to Eq. (34) with the replacement $\mathcal{M} \rightarrow \mathcal{M}\left(\lambda_{i}, \lambda_{f}\right)$ (of course Eq. (40) also holds at the level of unpolarized matrix elements). Also in this case, the scheme dependence of the one loop electroweak corrections is cancelled by the corresponding scheme dependence of the QED integrated dipoles.

\section{REAL EMISSION AND SUBTRACTION PROCEDURES}

Conceptually the calculation of the real emission processes and the appropriate subtraction terms for the cancellation of QED infrared singularities in numerical simulations is very similar to the case of QCD calculations. Many concepts and methods that were originally developed for QCD calculations can easily be adapted to electroweak computations. The additional radiation of a photon is a source of infrared singularities in regions of phase space where the photon becomes either soft or collinear to other charged massless particles. For sufficiently inclusive observables, the KLN theorem [202, 203] guarantees the cancellation of infrared singularities from final state particles against their counterparts from the virtual contributions. Collinear initial state singularities have to be either absorbed into the parton distribution functions, or these singularities have to be regulated using the physical mass of the incoming particles. The corresponding logarithms of the masses are a potential source of large corrections.

In the context of one loop electroweak calculations, the real contributions are basically defined as the real QED corrections to the process under consideration. Nevertheless, also the radiation of an additional $W / Z$ boson would contribute at the same order in perturbation theory. In the literature, the extra emission of additional $W$ and $Z$ bosons usually is not included in the computation of real $\mathcal{O}(\alpha)$ corrections for two reasons. First of all the gauge 
bosons decay, so that in principle they lead to final states which are not degenerate with the LO ones. The second reason is that real purely weak corrections are always finite: in fact, even in the Sudakov regime, where the corrections are dominated by the Sudakov logarithms which are the IR limit of virtual weak corrections, the gauge boson masses act as physical IR regulators. However, in those kinematical regions where the Sudakov corrections become large, the effect of the partially compensating radiation of real gauge bosons may lead to significant positive contributions. In Refs. [90 92, 94, 135, 139, 204 208] the issue of real weak boson emission has been addressed in analogy with QED or QCD: all the diagrams obtained from the LO ones with the emission of an additional $W$ or $Z$ boson are considered as real corrections, the additional gauge boson is produced on-shell and integrated over the full phase space. The result is a significant cancellation between real and virtual corrections, which however may be incomplete due to the incomplete average on the isospin of the initial state particles [243] (Bloch Nordsieck violating effects [90 92]). Real weak boson emission processes have been considered from a more phenomenological point of view in Refs. [97, 207, 209, 210]: the additional gauge bosons decay and they are included in the real corrections only when the final states are degenerate with the LO ones. Also in this case, the cancellation between real and virtual weak corrections is only partial and strongly dependent on the event selection under consideration. Recently, two different implementations of multiple weak boson emission processes in the context of parton showers have been presented in Refs. [211] and [212].

From the theoretical point of view the real emission of a photon can be handled in the same way as it is done in QCD, namely by defining jets as infrared safe observables, and a jet may or may not contain a photon. A clean way of doing that is the 'democratic clustering' approach [213] where all the partonic final states (hadrons and photons) are treated on equal footing. In that sense the photon is then treated fully inclusively and the results are infrared safe. For many final states however the presence of an additional photon can experimentally be tagged and therefore be distinguished from the original Born process. For instance a muon being collinear to a photon can be disentangled as they end up in different parts of the detector. From the theoretical point of view, this implies that the photon is not treated fully inclusively but parts of the collinear singularity is cut away. This leads to the problem that the collinear singularity in the virtual corrections is not fully canceled by the corresponding one in the real emission. As the muon mass acts as physical cut-off for the collinear singularities, the only effect 
of the miscancellation is a large QED correction. However, the incomplete cancellation would be an issue in the case of the photon radiation off a massless particle. There are two options to handle this problem. The first starts from the observation that a photon can either be produced in the hard scattering, i.e. it can be treated perturbatively, or it occurs much later at lower energies, when hadrons are created and decay into more stable hadrons. This happens in an energy regime that is non-perturbative and which can be described by the photon fragmentation function [213]. The photon fragmentation function is an observable that has to be measured experimentally, similarly to the parton distribution functions. And in the same way as initial state collinear singularities can be absorbed into the parton distribution functions, the part of the collinear singularity that is not canceled by the virtual corrections can be absorbed into the photon fragmentation function. The second option to deal with this incomplete cancellation is to (also experimentally) only cut away finite pieces such that the cancellation of the singularity remains unspoiled. This can be achieved by reducing the allowed amount of photonic energy within the jet as a function of the R-separation between photon and tagged particle such that the photon energy vanishes in the limit of collinearity [214]. We note that this problems also occur in fixed order QCD calculations where photons are present.

Additional complications arise from the fact that for a general process one cannot distinguish between QCD corrections and electroweak corrections but all contributions at a given order in $\alpha$ and $\alpha_{s}$ have to be taken into account. For the real emission this includes contributions from a gluon exchange between the interference of a QCD Born diagram with an electroweak Born diagram. It is worth to stress that these contributions are therefore not the absolute squared of an amplitude but just the interference term between two different types of amplitudes. They have different properties concerning their color -but also their kinematical- structure compared to standard tree-level like contributions. These terms can be handled with standard methods from QCD and we refer to section IV] for a concrete example, nevertheless their automated generation is in general not trivial (but it is within the reach of the tools dealing with automated calculations). 


\section{ELECTROWEAK CORRECTIONS TO $W^{+}$PLUS TWO JETS}

In the last part of this review we show a computation that has been constructed in a quasi automated way interfacing the GoSAM and MADDIPOLE programs. We calculate the electroweak NLO corrections to the production of a $W^{+}$boson in association with two jets, which has also been calculated in Ref. [138]. In the present calculation we treat the $W$-boson as a stable particle. Although this might be of limited interest from a purely phenomenological point of view, the decay of the $W$ does not add any fundamental complication to the calculation which is not already present in the approach of a stable $W$-boson. Of course, in the case of an off-shell $W$ production, the calculation should be rephrased in the context of the complex mass scheme; moreover, the IR subtraction procedure might be modified in order to have an efficient integration of real matrix elements plus local counterterms also in the presence of resonances [215, 216]. The complete NLO computation should include the decay of the gauge boson together with the contributions of photon induced processes. For simplicity, we neglect both these contributions although they could be computed in the same framework of our calculation. By the way, these contributions will not affect significantly the inclusive results, but in particular the photon initiated processes can be important in the tails of distributions: indeed, they could well be the subject of a dedicated phenomenological study [244]. For all these reasons, we stress that in this review the process $W+2$ jets serves as a proof of concept where one faces many subtleties of an electroweak computation while at the same time minimizing the necessary computational efforts. In fact, in many aspects this process is identical to the calculation of the dijet process, which has been presented in Ref. [119].

\section{A. Computational setup}

The starting point is a Born contribution of the order $\mathcal{O}\left(\alpha_{s}^{2} \alpha\right)$. At this order in the coupling constants two classes of diagrams contribute to the Born process: the ones with two external quarks (with two additional gluons either radiated off the quarks or produced via gluon splitting) and the ones with four quarks involving a gluon exchange between the two fermionic currents. In principle there would be also a Born contribution of the order $\mathcal{O}\left(\alpha^{3}\right)$ involving only diagrams with four quarks where the internal gluon is replaced by a photon or a massive gauge boson. 

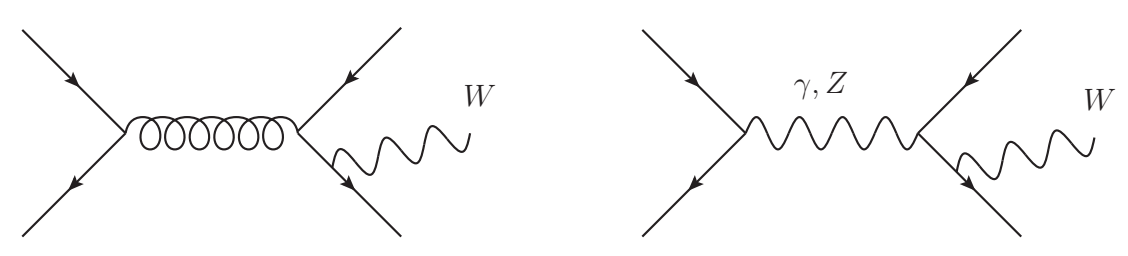

FIG. 3: Example diagrams for a QCD Born (left diagram) and an electroweak Born (right diagram). At leading order only the left diagrams are taken into account.

However, for the NLO EW corrections to $W+2$ jets, one can safely neglect the $\mathcal{O}\left(\alpha^{3}\right)$ underlying Born: it would lead to $\mathcal{O}\left(\alpha^{4}\right)$ contributions which will be much smaller than the $\mathcal{O}\left(\alpha_{s}^{2} \alpha^{2}\right)$ ones due to the different sizes of the coupling constants. Two sample diagrams are depicted in Fig. 3 .

The fact that the $W$-boson is treated as a stable particle raises an additional complication at one loop. In fact, the building blocks of the calculation involve diagrams with internal massive propagators that can give rise to singularities when the momentum flowing in these propagators approaches the on-shell limit. On one hand these singularities could be avoided by means of the complex mass scheme, which would imply that the on-shell prescription for the process $W+2$ jets should be abandoned. On the other hand, a naive inclusion of the widths in the massive propagators would lead to inconsistencies because of the different treatment of the external and the internal $W$ bosons (in particular, the soft singularities corresponding to the $W-\gamma$ splitting would be turned into logarithms of the widths). However, in the process $W+2$ jets at $\mathcal{O}\left(\alpha_{s}^{2} \alpha^{2}\right)$ these potentially problematic diagrams are interfered with a QCD Born that does not involve internal massive particles. This means that these terms do not give rise to physical resonances, however they are only integrable as principal value integrals. For a numerical Monte Carlo integration this is however problematic as it would simply try to integrate over a singular region.

As discussed in Ref. [138], one possible solution is to introduce a regulator width $\Gamma$ in those pseudo-resonant diagrams that are not associated with IR singularities, so that the remaining diagrams are free of pseudo-resonances. Though this procedure technically breaks gauge invariance, the violation of gauge invariance is of order $\Gamma / M$. For the one loop contribution we adopt a different method that is based on a local K-factor. For each phase space point we require that the finite part of the one loop contribution normalized to the underlying QCD Born (i.e. 
a Born that is free of resonances) has to be smaller than a certain value. The advantage of this approach is that one does not have to introduce a width for only specific diagrams. Nevertheless it can be related to the method of using a regulator width: in fact, a large K-factor is obtained if the internal particle is close to being on-shell and the K-factor effectively cuts the contribution off if the particle is too close to being on-shell. One can roughly estimate the width corresponding to a given $\mathrm{K}$-factor by comparing the massive propagator to a massless one, requiring that

$$
\frac{p^{2}}{p^{2}-m^{2}}<K
$$

and solving Eq. (41) for $p$ for a given value of $K$. This way one obtains the minimally allowed distance from the mass $m$. It is worth noting that the procedure in Eq. (41) is only an approximation, as in general many other aspects enter in the K-factor. As a default we used a value of 1000 for the K-factor, but we varied its value from one hundred to one thousand and found that the results for the different values of the K-factor agree within their statistical errors. To further reduce numerical instabilities we generated the diagrams with a Higgs exchange separately and used an integration channel that resembles exactly the peak structure of these diagrams. This may serve as an additional strategy to improve numerical convergence for parton level results: loop diagrams from which one expects different peak structures can be generated separately and then integrated with a dedicated parametrisation of the phase space.

For the subtraction terms consisting of a gluon exchange between an EW Born and a QCD Born as illustrated in Fig. 4 one can in principle use the same method, which is however not very convenient, as one has to perform this check for each subtraction term with its remapped kinematics. And it also requires to calculate the matrix element of the QCD amplitude. Therefore we adopt the method of Ref. [138] and used a regulating width for the internal $W$ - and $Z$-bosons. We varied this with between 0.1 and $1 \mathrm{GeV}$ and found agreement between the results within their statistical uncertainties.

\section{Virtual corrections}

For this process we have to distinguish two different types of virtual corrections. Starting from the QCD like born structure we have to calculate the one-loop electroweak corrections leading to a contribution of $\mathcal{O}\left(\alpha_{s}^{2} \alpha^{2}\right)$. However this order in perturbation theory can also 
be achieved by interfering a loop amplitude of $\mathcal{O}\left(\alpha_{s}^{2} \alpha^{1 / 2}\right)$ with a pure electroweak tree-level amplitude of $\mathcal{O}\left(\alpha^{3 / 2}\right)$. This contribution can be understood as an interference term between an electroweak tree-level amplitude with a QCD one-loop amplitude originating from the QCD born. The two different contributions are illustrated in Fig. 5. The lower two plots show an example diagram for the two cases. The left diagram contains a loop with both a gluon and a photon (or a weak gauge boson) in the loop. This is a good example that the distinction between QCD corrections and electroweak corrections does not make sense here, as this diagram can be regarded to be a QCD correction to an electroweak Born as well as an electroweak correction to a QCD born. This also means that these kinds of diagrams might contain singularities from both interactions, QCD and electroweak. The loop on the right hand is a pure QCD correction and therefore only contains QCD singularities.

Simply speaking this situation always arises in the case where at tree-level the desired final state can be obtained in two different ways, either with a QCD Born or with an electroweak Born. The two contributions can be calculated independently, however, only the sum of the two yields a physically meaningful result and one has to sum up all the terms that contribute to a given order in perturbation theory at next-to-leading order, both in $\alpha_{s}$ and $\alpha$.

There is one subtle difference between the two contributions. In the first case one starts from a QCD born amplitude with a given order in $\alpha$ and $\alpha_{s}$ and the one-loop amplitude just adds another factor of $\alpha$ while keeping the original order in $\alpha_{s}$. This is also the situation one encounters performing QCD corrections and this is in some sense the standard way. The second contribution however is different. Starting from an electroweak born amplitude, the corresponding one-loop amplitude is two powers higher in $\alpha_{s}$ but also one power lower in $\alpha$. In view of automating electroweak calculations and combining different tools, in particular one-loop providers (OLP) and Monte Carlo tools (MC), this is a special case that is not included in the currently used standard interface [217, 218]. Therefore an appropriate extension of the existing interface would be highly desirable.

In this calculation the virtual amplitudes are generated with GoSAM [21, 22]. The GoSAM framework is based on an algebraic generation of $D$-dimensional integrands using a Feynman diagrammatic approach, employing QGRAF [219] and FORM [220, 221] for the diagram gen- 

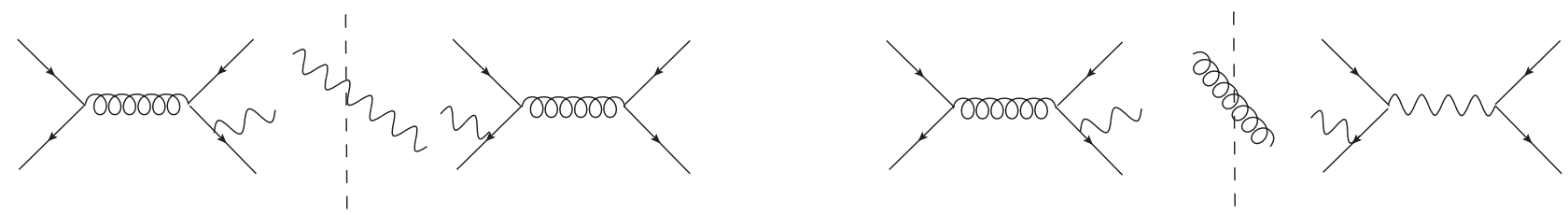

FIG. 4: Schematical representation of the two different kinds of insertion operators, representing QED singularities (l.h.s.) and QCD singularities (r.h.s.). This also illustrates the two different types of real emission contributions.

eration, and SPINNEY [222], HAgGies [223] and FORM to write an optimized Fortran output. For the reduction of the tensor integrals we used NinJA [224 226], a package for the integrand reduction via Laurent expansion. One can however also use different reduction techniques such as integrand reduction via the OPP method [13, 227, 228] as implemented in SAMURAI [15] or one can use methods of tensor reduction as the ones contained in Golem95 [229 232]. The remaining scalar integrals have been evaluated using OnELoop [233]. All these methods and tools have originally been developed for the case of QCD one-loop corrections. However for none of them it matters whether one uses them for QCD corrections or for electroweak corrections. The generation of the diagrams, and the reduction of the tensor integrals or the integrand reduction respectively rely on general properties that do not depend on the type of the interaction.

As GoSAm allows to specify the orders of tree-level amplitude and one-loop amplitude separately, i.e. does not rely on the fact that the one-loop amplitude is just one order higher in the coupling constant, it is straightforward to obtain the two different virtual contributions. We have extended the GoSAm framework to incorporate the electroweak counter terms as well as the electroweak infrared insertion operator.

The advantage of the inclusion of the insertion operator is twofold. In GoSAm the agreement of the poles is the first level of the trigger for numerical instabilities. The numerical structure of the insertion operator is very simple and it is proportional to the Born. However on the side of the one-loop amplitude this simple results is obtained in a highly non-trivial way. Numerical instabilities can be detected as deviations from the correct coefficient of the pole terms. This can then either be used to trigger some kind of rescue system or it can be used to make quantitative estimation on the accuracy of the obtained result. As the pole cancellation only works for the 

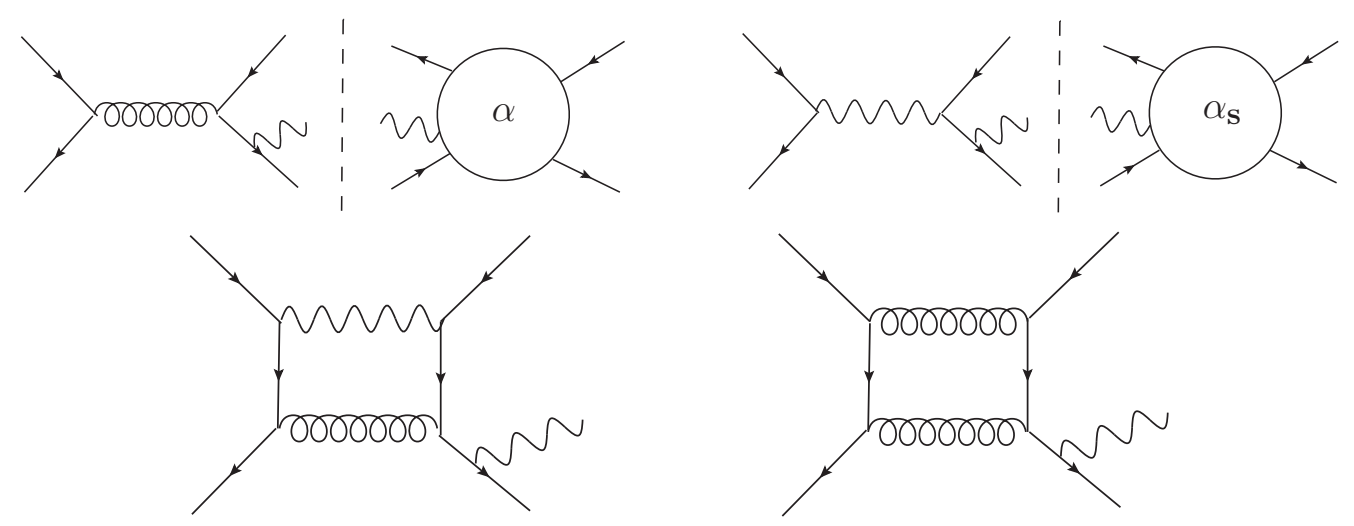

FIG. 5: Different virtual contributions. In the upper row the left plot denotes the interference between a QCD tree-level diagram with an electroweak one-loop diagram, the right plot displays the interference between an electroweak tree-level diagram and a QCD one-loop diagram. As the two pieces contribute at the same order both have to be taken into account. The lower row shows an example diagram for each of the two types of one-loop amplitudes.

renormalized amplitude this is also an important check for the electroweak counter terms, which are new in the GoSAm framework. Especially for these types of processes, where one has different virtual contributions, the check of this pole cancellation is very important as the cancellation only takes place when combining electroweak and QCD one loop contributions.

We should stress that the formulas for the insertion operator in Eq. (21) are only valid for contributions from the l.h.s of Fig. 5. They are obtained by photon insertion between the underlying QCD amplitudes, i.e. the insertion operator only covers the QED singularities of these diagrams. It does not cover any singularity of QCD origin which are contained in the diagrams of the r.h.s. of Fig. 5. Therefore, in general one does not find pole cancellation between the two terms in Fig. 5 and the QED insertion operator. The singularities from contributions stemming from the r.h.s of Fig. 5 are of purely QCD origin. This means that we need to apply a QCD insertion operator. In contrary to normal QCD corrections, the born-like matrix element is given by the interference term between a QCD Born amplitude and an electroweak Born amplitude with an additional gluon insertion that modifies the color structure of the interference term (as it does in normal QCD). The singularities from this insertion operator will then cancel against the QCD singularities from one-loop contributions of both 1.h.s. and r.h.s diagrams in Fig. 5. The two different types of insertion operators are illustrated in Fig. 4. 
For the production of a $W$-boson in association with jets the number of processes that need to be generated can be reduced to:

$$
\begin{gathered}
\bar{u} u \rightarrow W^{+} \bar{u} d \\
u \bar{d} \rightarrow W^{+} c \bar{c} \\
u \bar{d} \rightarrow W^{+} s \bar{s} \\
u \bar{d} \rightarrow W^{+} g g \\
\bar{u} u \rightarrow W^{+} \bar{c} s \\
\bar{d} d \rightarrow W^{+} \bar{c} s \\
u \bar{d} \rightarrow W^{+} d \bar{d} .
\end{gathered}
$$

Note that for the process $u \bar{d} \rightarrow W^{+} g g$ there exists no underlying electroweak born, hence this process does not have a QCD one-loop contribution.

We have checked the pole cancellation mentioned above for all the independent subprocesses. All other processes can be obtained by crossing. Besides the renormalization of the electroweak sector this type of process also requires the renormalization of QCD singularities. They arise from the QCD loop diagrams that are interfered with an electroweak born process. The renormalization procedure itself is straightforward and identical to a conventional QCD calculation, however the interpretation is a bit different. The underlying born that needs to be renormalized is an interference term between the QCD born and the electroweak born rather than the absolute square of an amplitude at a given order in $\alpha_{s}$.

\section{Regularization scheme dependence}

In the GoSAm framework dimensional reduction is the more appealing approach as one deals with a simplified $D$-dimensional Dirac algebra and does not need to take care about additional renormalization terms for $\gamma_{5}$. This typically leads to more compact expressions and hence is the method of choice. It is worth mentioning that for the given process, the scheme dependence for the QCD loops is proportional to the interference term between the QCD Born and the electroweak Born, with an additional modified color factor. For subprocesses that only have one color structure the scheme dependence vanishes. This is however not a general feature and does not hold for more than one color factor. 


\section{Real emission}

The real emission contributions contain processes based on an underlying QCD Born with an additional photon. We neglect additional radiation of massive electroweak gauge bosons. As discussed above, a second contribution stems from the radiation of an additional gluon from an underlying electroweak Born amplitude, interfered with a QCD Born amplitude. This contribution is necessary to cancel the QCD like singularities from the virtual corrections. All real emission contributions and corresponding subtraction terms for both QCD and QED radiation have been generated with MADGRAPH 4 [234, 235] and MADDIPOLE [44 46], containing subtraction terms based on the dipole formalism as developed in [153, 196]. The QED version of MAdDipole has been extended to cover the photon radiation off the $W$-boson. The integration over tree-level and real emission phase space has been performed using MADEvEnT [236]. The inclusion of the virtual contributions has been obtained using the BLHA interface, which has been adapted to be usable for electroweak corrections.

\section{Parameters}

As a consistent set of input parameters we use $M_{W}, M_{Z}$ and $G_{\mu}$ with

$$
M_{W}=80.398 \mathrm{GeV}, \quad M_{Z}=91.1876 \mathrm{GeV}, \quad G_{\mu}=1.1663787 \cdot 10^{-5} \mathrm{GeV}^{-2},
$$

while $\alpha$ is a derived quantity:

$$
\alpha_{G_{\mu}}=\frac{\sqrt{2} G_{\mu} M_{W}^{2}}{\pi}\left(1-\frac{M_{W}^{2}}{M_{Z}^{2}}\right) .
$$

The $G_{\mu}$ input scheme avoids large logarithms involving light fermion masses originating from the running of $\alpha$ from the Thomson limit to the electroweak scale [119, 171]. As these logarithms are effectively included in the definition of the coupling constant, they have to be subtracted from electric charge renormalization counterterm with the following replacement [49, 119, 171]:

$$
\left.\delta Z^{e}\right|_{G_{\mu}}=\left.\delta Z^{e}\right|_{\alpha(0)}-\frac{1}{2} \Delta r
$$

where $\left.\delta Z^{e}\right|_{\alpha(0)}$ is defined in Eq. (10) while $\Delta r$ summarizes the radiative corrections to muon decay [183 185] apart from the QED corrections which coincide with those of the Fermi model. Due to the real emission of the photon, which happens at $q^{2}=0$ one power of the coupling 
constant $\alpha$ is taken to be as $\alpha=\alpha(0)=1 / 137.035999074$. Moreover, the one loop renormalization is a function of the Higgs and the top quark mass. In our numerical studies we used the values:

$$
\begin{array}{ll}
m_{e}=510.99892 \mathrm{KeV}, & m_{\mu}=105.658369 \mathrm{MeV}, \quad m_{\tau}=1.77699 \mathrm{GeV}, \\
m_{t}=172.9 \mathrm{GeV}, & M_{H}=120 \mathrm{GeV} .
\end{array}
$$

As the $W$ is treated as a stable particle in this calculation, no complex mass scheme is applied here.

Renormalization and factorization scale are both set to $M_{W}$, the center of mass energy is chosen to be $14 \mathrm{TeV}$. As this is a calculation which is leading order in QCD we use the MSTW2008lo pdf set. The CKM matrix has been chosen to be a diagonal unit matrix. In order to set cuts in an infrared safe way we use the following approach of democratic clustering: all particles, including the $W$-boson are passed through the jet algorithm and clustered according to an anti-kt algorithm [237, 238] contained in the FASTJET package [239]. In a second step we however require that the $W$-boson as well as two jets containing a QCD parton are tagged. This ensures that we are also infrared safe from a QCD point of view. The resulting jets need to fulfill the following basic cuts:

$$
p_{T}>20 \mathrm{GeV}, \quad|\eta|<4.4, \quad \Delta R>0.4
$$

This is in particular also true for the $W$-boson.

\section{B. Numerical Results}

With the set of parameters stated above we find a total cross section at leading order of

$$
\sigma_{t o t, L O}=10873 \pm 6 \mathrm{pb}
$$

As expected, the electroweak NLO corrections only have a small impact on the total cross section. Their contribution is negative and we find a total cross section of

$$
\sigma_{t o t, N L O}=10789 \pm 6 \mathrm{pb}
$$

Therefore we find a correction of $\sim 1 \%$ to the total cross section. The errors in both eqs. (48) and (49) represent the $1 \sigma$ Monte Carlo integration error. 

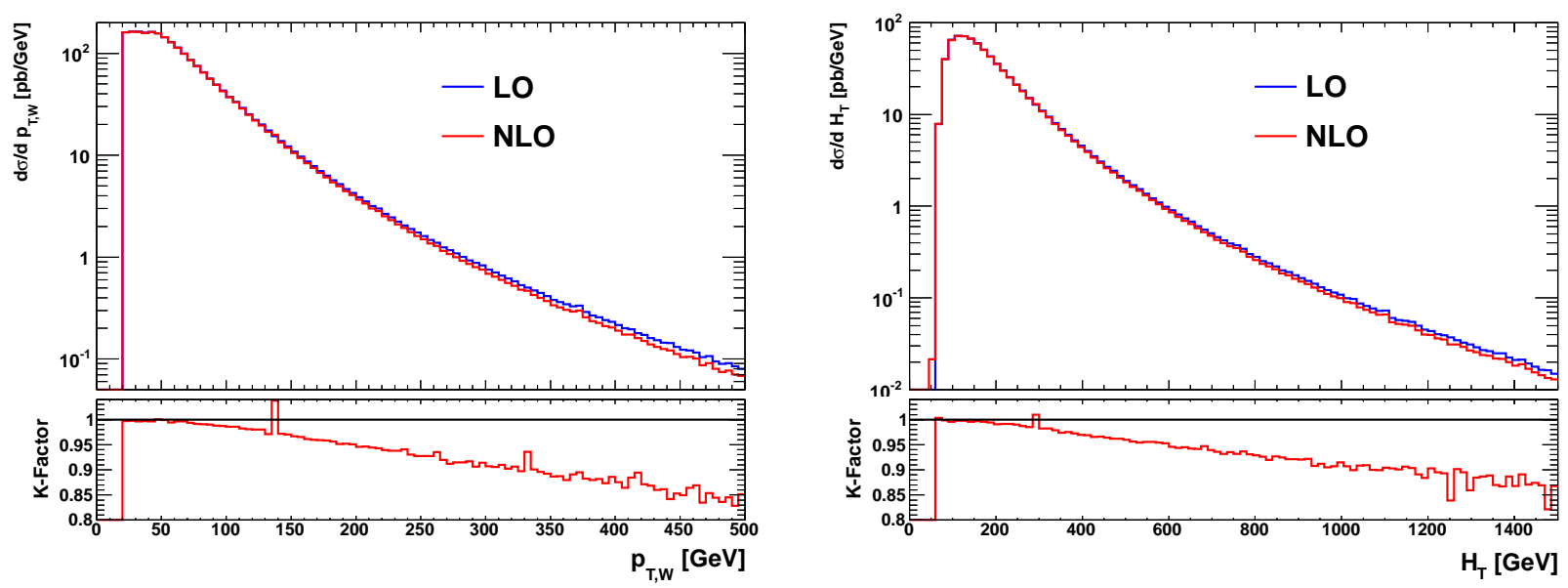

FIG. 6: Differential distribution of the transverse momentum of the $W$-boson (left plot) and the $H_{T}$ distribution (right plot).

More interesting than the total cross section are however differential distributions as one expects deviations stemming from contributions of large logarithms of the $W$ mass (see e.g. [240]). In Fig. 6 we show the transverse momentum of the $W$ boson. Although the impact in the low energy region is negligible we do not obtain a constant K-factor but we see a substantial decrease of the cross section in the region where the transverse momentum of the $W$ is large compared to its mass. In this region electroweak corrections typically supersede QCD corrections leading to a $\sim 20 \%$ correction in the region of $500 \mathrm{GeV}$. A similar behavior is observed for the $H_{T}$ distribution, also leading to a $\sim 20 \%$ in the high energy tail of the distribution. This behavior stresses the importance of electroweak corrections at the LHC in the context of searches for new physics, as one would typically search for new physics in the high energy limit. For observables that are sensitive to Sudakov logarithms, like the transverse momenta or the $H_{T}$ distribution, the size of the electroweak corrections grows beyond the uncertainty from the QCD corrections in the tails of the distributions.

An interesting behavior can also be observed for the difference in rapidity between the $W$ and the jets $\Delta y_{j 1, W}$. Fig. (7) shows this observable for the leading jet, i.e. the jet with the highest transverse momentum. One observes a negative correction for small differences in rapidity, but a positive correction for large values of $\Delta y_{j 1, W}$ which are however not relevant for the total cross section, as the contribution to the total cross section from this region is small. The radiation of a particle with a high transverse momentum will more likely happen to take place in the central 


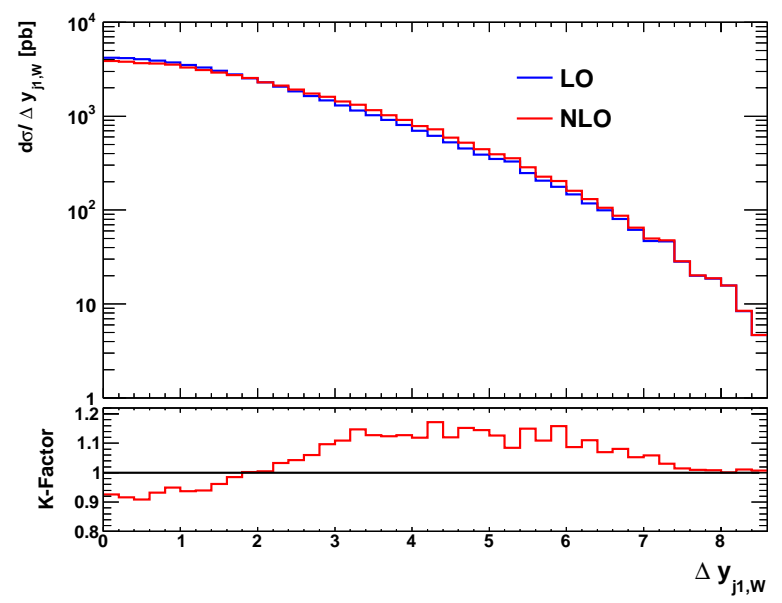

FIG. 7: Differential distribution for absolute value of the rapidity difference between the $W$ and the leading jet.

region, as one needs less energy to get the same transverse momentum as in a more longitudinal direction. A $W$-boson with a high transverse momentum has to recoil against the jets (or at least the leading jet). Therefore we expect the events with a high transverse momentum of the $W$ happening for small differences in rapidity, which explains the negative next-to-leading order corrections in this region. Turning this argument around we expect events with large differences in rapidity to come with smaller values of the transverse momentum. This leaves more phase space to additional radiation which comes with a positive contribution and hence shifting the NLO corrections to positive values.

The same behavior is also found for the rapidity distribution of the $W$ which is shown in the left plot of Fig. 8, although the rapidity distribution to a larger extents mixes events with different transverse momenta and therefore the effect is much less pronounced. Generally speaking we do not expect visible effects of electroweak NLO corrections for observables where the events with high transverse momentum of the $W$ are evenly distributed as a function of the observable under consideration. One such example is shown in the right plot of Fig. 8, where the difference in the azimuthal angle between the two leading jets is displayed. In that case we obtain a flat K-factor yielding a small negative correction at next-to-leading order. 

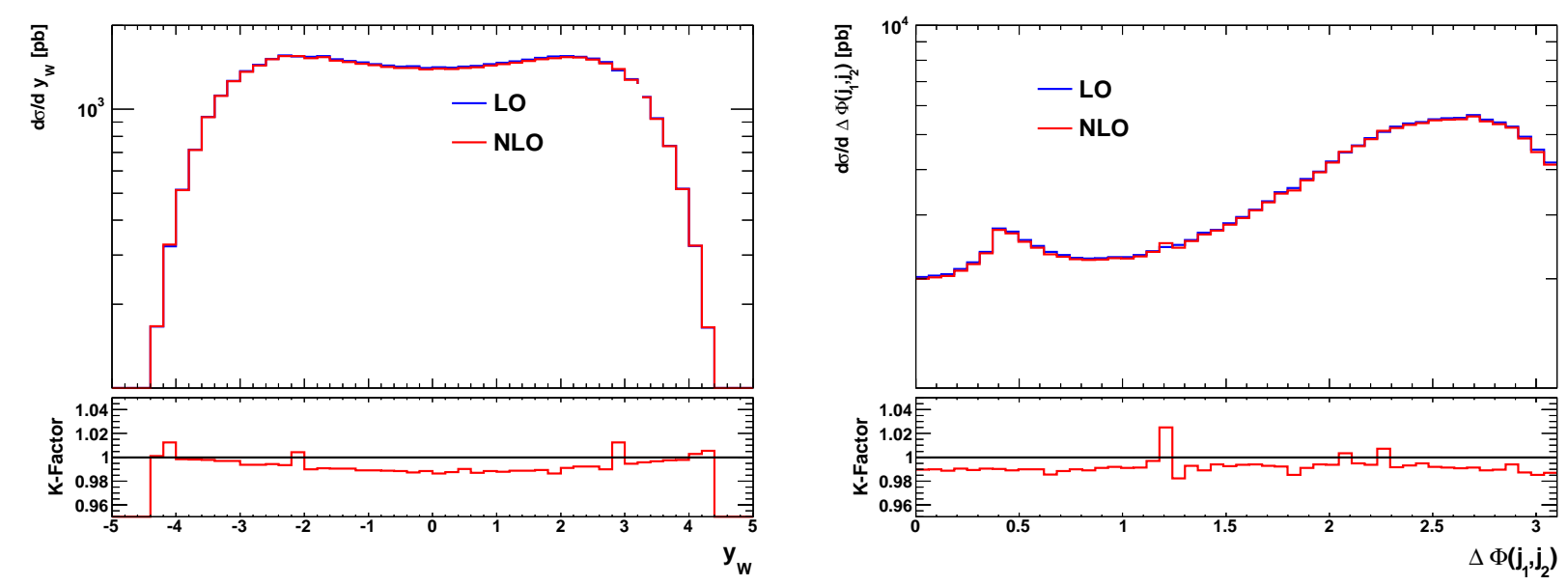

FIG. 8: Differential distribution of the rapidity of the $W$-boson (left plot) and the difference of the azimuthal angles of the two leading jets (right plot).

\section{Virtual amplitudes for single phase space point}

In this section we give the results for all the ingredients that enter the virtual contribution for a single phase space point reported in Table I. The regularization scheme is DRED, the couplings $g_{s}$ and $e$ are set to one for simplicity and all the results in the tables do not contain the overall factor $\alpha_{(s)} / 2 \pi$. The other parameters are set as stated above. The notation is as follows: Born-QCD denotes the tree-level matrix element squared that is of the order $\mathcal{O}\left(\alpha_{s}^{2} \alpha\right)$, while Born-EW denotes the tree-level matrix element squared of the order $\mathcal{O}\left(\alpha^{3}\right)$ [245]. The first line in the tables gives the contribution of the unrenormalized electroweak corrections, the second line the corresponding counter terms. The third line denotes the unrenormalized contribution of QCD-loops interfered with the electroweak tree-level amplitudes, followed by QCD renormalization terms. The last two lines then denote the contributions from the electroweak and QCD insertion operators. 


\begin{tabular}{cccc}
$E$ & $p_{x}$ & $p_{y}$ & $p_{z}$ \\
\hline \hline 250.0 & 0.0 & 0.0 & 250.0 \\
250.0 & 0.0 & 0.0 & -250.0
\end{tabular}

$111.48897463938037 \quad 56.330449672673069 \quad-24.583961957306506 \quad 46.781003797444882$

$207.38546864044983-167.21520572166636-118.01342209209260-33.475958310305167$

$\begin{array}{lllll}181.12555672016978 & 110.88475604899321 & 142.59738404939910 & -13.305045487139747\end{array}$

TABLE I: Phase space point where all the relevant amplitudes of Eq. (42) have been evaluated.

$$
\text { 1. } \bar{u} u \rightarrow W^{+} \bar{u} d
$$

Born-QCD: 0.3331176339143377E -03
\begin{tabular}{c|r|r|r|} 
Process & Born-EW: 0.1159155705277286E -02$)$ \\
& $1 / \epsilon^{2}$ & $\bar{u} u \rightarrow W^{+} \bar{u} d$ \\
EW-Loop unren. & $0.4515129528621876 \mathrm{E}-03$ & $0.5179344797596786 \mathrm{E}-02$ & $-0.4048755402433699 \mathrm{E}-02$ \\
EW-Ren. & 0 & $-0.4682725341929206 \mathrm{E}-02$ & $-0.6484221636837220 \mathrm{E}-03$ \\
QCD-Loop unren. & $0.9326828685162938 \mathrm{E}-03$ & $-0.2347576391436467 \mathrm{E}-02$ & $0.1872325091832032 \mathrm{E}-02$ \\
QCD-Ren. & 0 & $0.1224146264927649 \mathrm{E}-02$ & $0.8851406118179909 \mathrm{E}-05$ \\
I-EW & $0.4811699156540433 \mathrm{E}-03$ & $-0.1471889938882662 \mathrm{E}-02$ & - \\
I-QCD & $-0.1865365737032609 \mathrm{E}-02$ & $0.2098700609724008 \mathrm{E}-02$ & - \\
\hline$\sum$ & $-0.8 \mathrm{E}-16$ & $0.1 \mathrm{E}-15$ & $-0.2815845104231514 \mathrm{E}-02$
\end{tabular} 
2. $u \bar{d} \rightarrow W^{+} c \bar{c}$

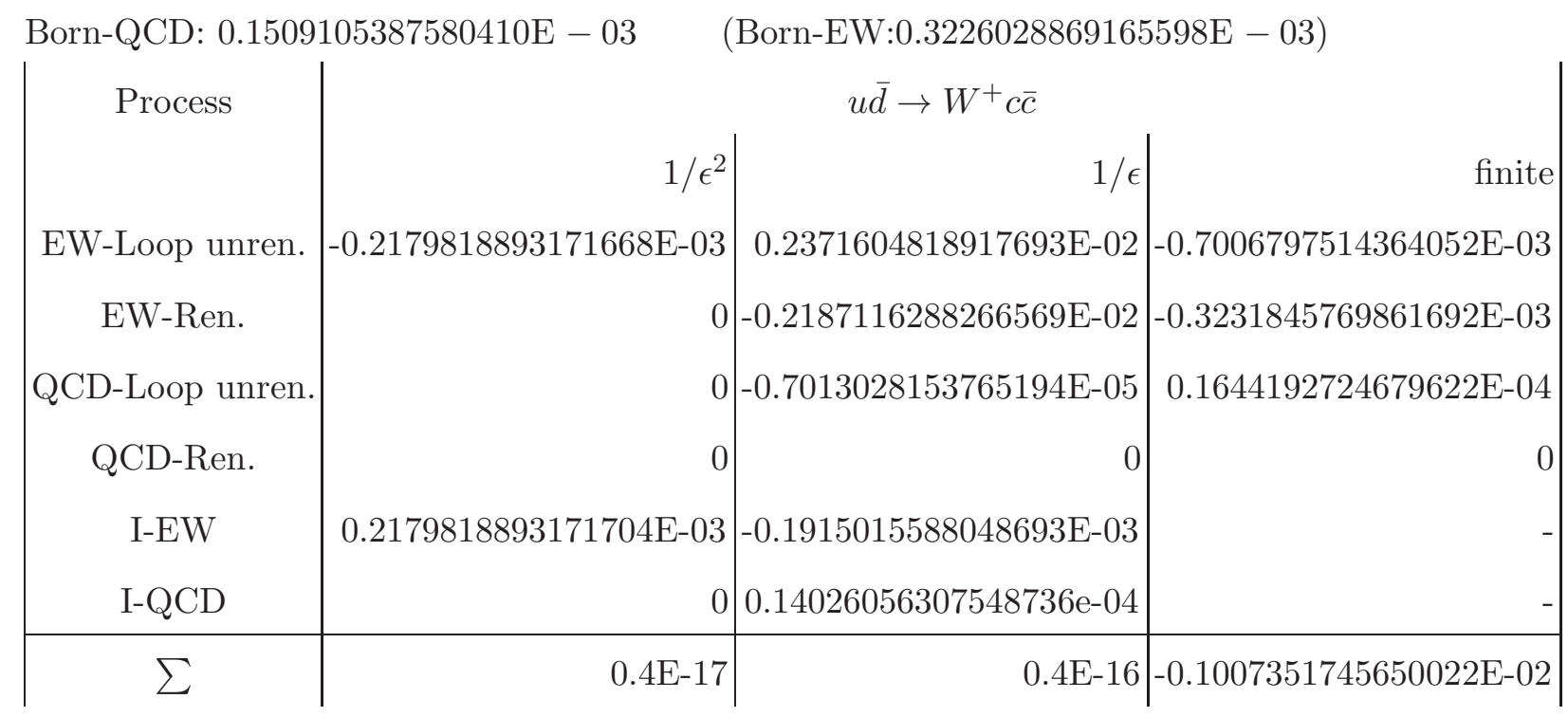

3. $u \bar{d} \rightarrow W^{+} s \bar{s}$

Born-QCD: 0.1509105387580410E - 03 (Born-EW: 0.1683155172046103E - 03)

\begin{tabular}{|c|r|r|r|} 
Process & \multicolumn{3}{|r|}{$u \bar{d} \rightarrow W^{+} s \bar{s}$} \\
EW-Loop unren. & $-0.1173748634784783 \mathrm{E}-03$ & $0.2359759858112650 \mathrm{E}-02$ & $-0.8847947525100161 \mathrm{E}-03$ \\
EW-Ren. & 0 & $-0.2201404574690460 \mathrm{E}-02$ & $-0.3267300831621827 \mathrm{E}-03$ \\
QCD-Loop unren. & 0 & $0.1073376195468900 \mathrm{E}-04$ & $-0.8486634366399400 \mathrm{E}-04$ \\
QCD-Ren. & 0 & 0 & 0 \\
I-EW & $0.1173748634784764 \mathrm{E}-03$ & $-0.1476215214675483 \mathrm{E}-03$ & - \\
I-QCD & 0 & $-0.2146752390936322 \mathrm{e}-04$ & - \\
\hline$\sum$ & $-0.2 \mathrm{E}-17$ & $-0.3 \mathrm{E}-16$ & $-0.1296320523810437 \mathrm{E}-02$
\end{tabular} 


$$
\text { 4. } u \bar{d} \rightarrow W^{+} g g
$$

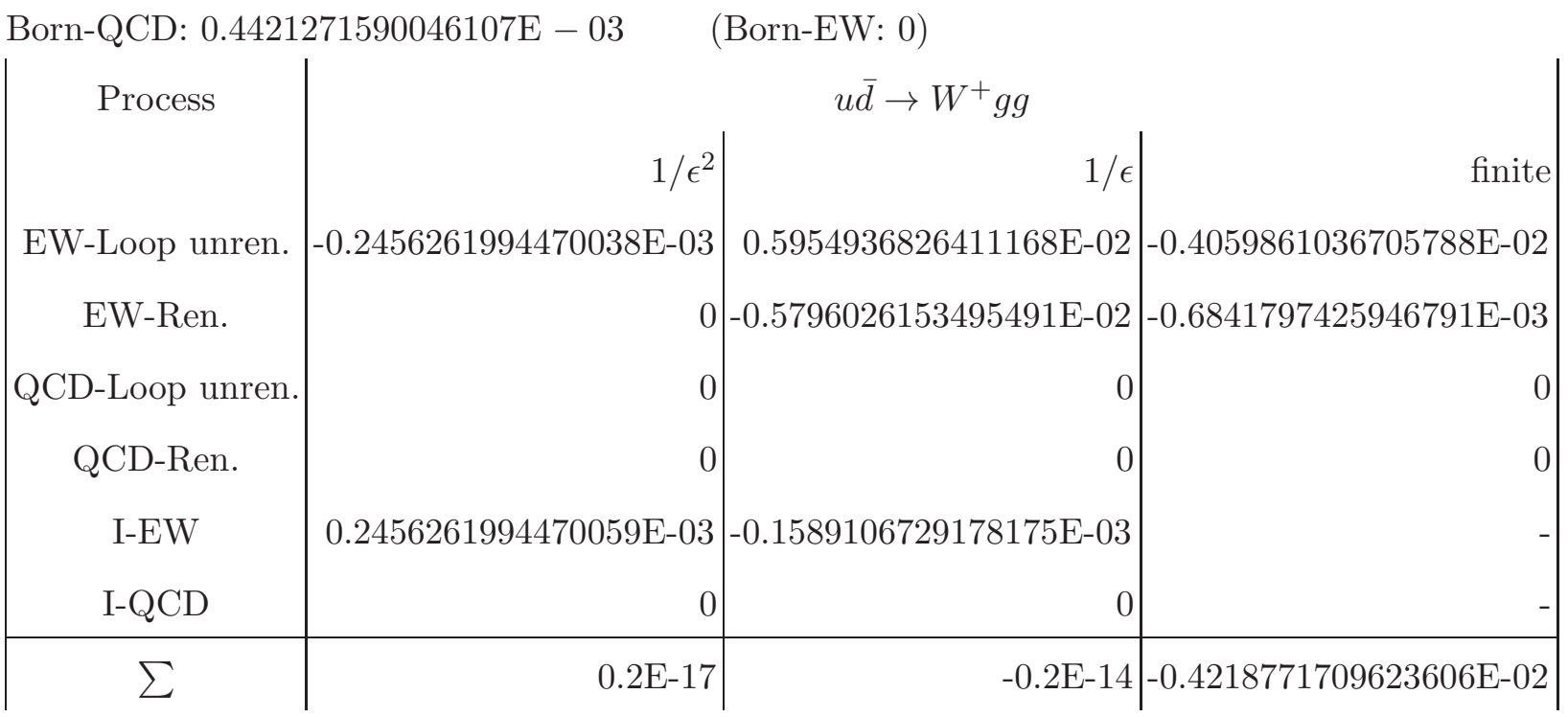

$$
\text { 5. } \quad \bar{u} u \rightarrow W^{+} \bar{c} s
$$

\begin{tabular}{|c|c|c|c|}
\hline \multicolumn{2}{|c|}{ Born-QCD: 0.5471920731180749E - 04} & \multicolumn{2}{|c|}{ (Born-EW: 0.1560261025155393E - 03) } \\
\hline Process & & $\bar{u} u \rightarrow W^{+} \bar{c} s$ & \\
\hline & $1 / \epsilon^{2}$ & $1 / \epsilon$ & finite \\
\hline EW-Loop unren. & $-0.7903885500595288 \mathrm{E}-04$ & $0.1039825482424979 \mathrm{E}-02$ & $0.6729212743821051 \mathrm{E}-03$ \\
\hline EW-Ren. & 0 & $-0.7933882016361721 \mathrm{E}-03$ & $-0.1173430614024200 \mathrm{E}-03$ \\
\hline QCD-Loop unren. & 0 & $0.4658874285701936 \mathrm{E}-05$ & $-0.6180475632648689 \mathrm{E}-04$ \\
\hline QCD-Ren. & 0 & & 0 \\
\hline $\mathrm{I}-\mathrm{EW}$ & $0.7903885500594414 \mathrm{E}-04$ & $-0.2417784065030774 \mathrm{E}-03$ & \\
\hline $\mathrm{I}-\mathrm{QCD}$ & 0 & $-0.9317748571408931 e-05$ & \\
\hline$\sum$ & $-0.9 \mathrm{E}-17$ & $0.2 \mathrm{E}-16$ & $0.49379907590015959 \mathrm{E}-03$ \\
\hline
\end{tabular}


6. $\quad \bar{d} d \rightarrow W^{+} \bar{c} s$

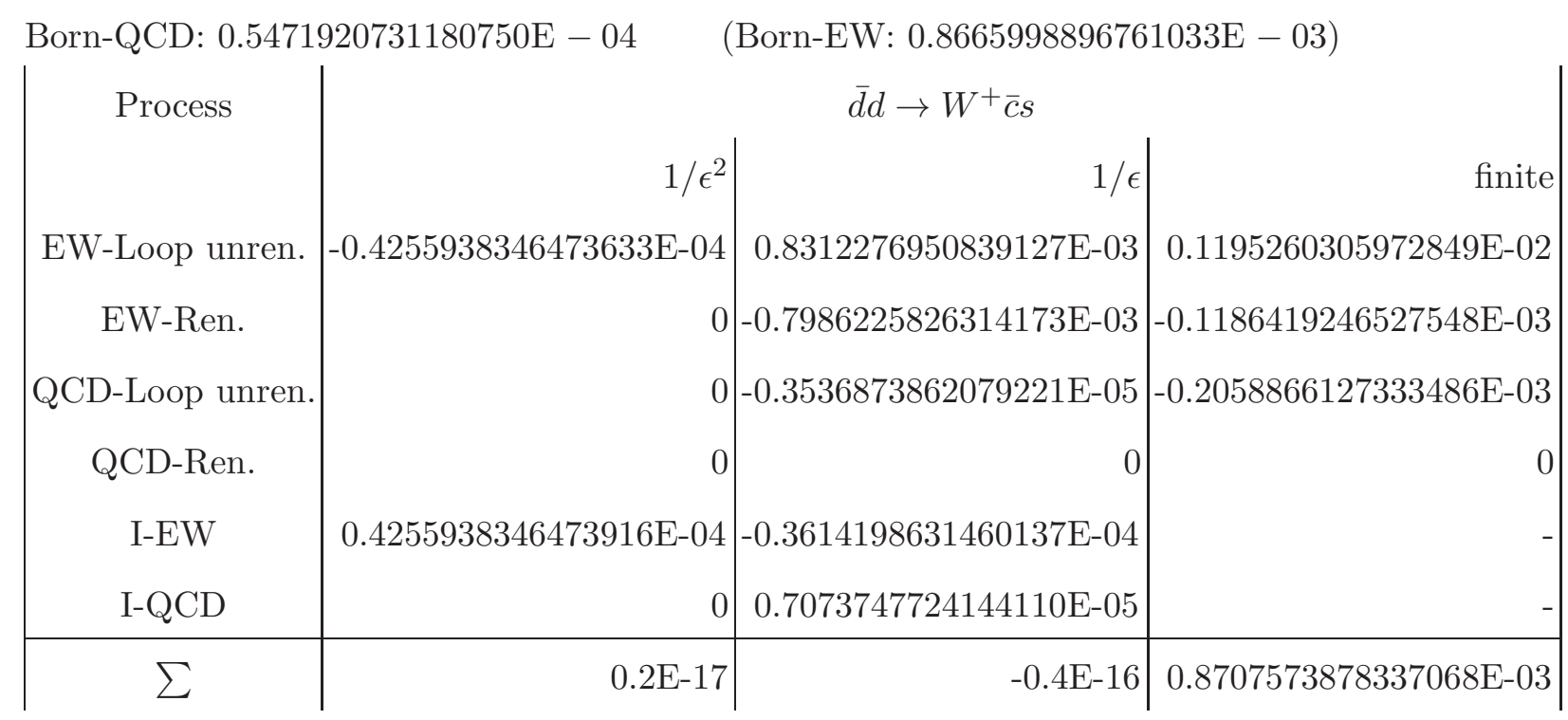

ๆ. $u \bar{d} \rightarrow W^{+} d \bar{d}$

Born-QCD: 0.8493852813137004E - 03

(Born-EW: 0.1939842768622516E - 02)

\begin{tabular}{|c|r|r|r|} 
Process & \multicolumn{3}{|r|}{$u \bar{d} \rightarrow W^{+} d \bar{d}$} \\
EW-Loop unren. & $0.1913723183724379 \mathrm{E}-02$ & $0.9838014169469896 \mathrm{E}-02$ & $-0.1220570133146914 \mathrm{E}-01$ \\
EW-Ren. & 0 & $-0.1197778947261055 \mathrm{E}-01$ & $-0.1665029533330621 \mathrm{E}-02$ \\
QCD-Loop unren. & $0.2574356180301684 \mathrm{E}-02$ & $-0.6257504923291653 \mathrm{E}-02$ & $0.1973036296675117 \mathrm{E}-02$ \\
QCD-Ren. & 0 & $0.3378842486646013 \mathrm{E}-02$ & $0.2443131831181329 \mathrm{E}-04$ \\
I-EW & $0.6606329965773225 \mathrm{E}-03$ & $-0.8308733675698238 \mathrm{E}-03$ & - \\
I-QCD & $-0.5148712360603448 \mathrm{E}-02$ & $0.5849311107356306 \mathrm{E}-02$ & - \\
\hline$\sum$ & $-0.6 \mathrm{E}-16$ & $0.2 \mathrm{E}-15$ & $-0.1187286557206206 \mathrm{E}-01$
\end{tabular}

\section{CONCLUSIONS}

The recent start-up of the LHC's Run 2 will allow to test the Standard Model through a set of new precision measurements. The search for new physics beyond the Standard Model is just started and new regions of phase space will be explored. The effect of the new physics 
might be large, but given the unique opportunity offered by the LHC physics program one has to look also for small deviations from the Standard Model. From the theoretical point of view this requires predictions based on precise and reliable computations whenever possible. This triggered the so called NLO revolution that was originally focused on providing NLO QCD corrections in an automated way. For many important processes like Higgs and top-quark production for instance the NLO corrections were not precise enough to meet the requirements. This lead to an ongoing active field of research namely pushing the limit further to NNLO QCD corrections. On the other hand also electroweak corrections will play an important role in a large number of searches. Although their impact on the total cross sections is typically rather small, they can lead to significant differences in distributions, in particular in the high energy tails. However it is exactly the high energy limit that is sensitive to new physics effects. Therefore, for a reliable comparison with LHC data from Run 2, the inclusion of electroweak higher order effects becomes inevitable for a large class of processes (also with large final state multiplicities). In this context, it is clear that fully automated tools for the computation of NLO electroweak corrections will play a crucial role at the LHC and at future hadron colliders.

We have presented an overview on the current status and recent development in the field of electroweak NLO corrections for LHC physics. In particular we focused on the topic of the automation of $\mathcal{O}(\alpha)$ calculations, which is a very active and fast developing area of research. We have summarized the existing tools and the phenomenological predictions that have been obtained for LHC relevant processes. Furthermore we have discussed some of the general issues, related to the calculation of either the virtual one loop corrections or the real emission contributions, that have to be faced in order to develop an automated tool for the computation of NLO electroweak corrections starting from already available programs such as GoSAM (for the generation of one loop amplitudes) and MADDIPOLE (for the subtraction of infrared singularities). We discussed the regularization scheme dependence of the virtual one loop amplitudes, the renormalization counterterms and infrared subtraction terms (at least for the specific case of the CDR and DRED schemes) and we provided the corresponding scheme transition rules that are required in order to add up consistently these three building blocks of the calculation. We also pointed out the non trivial aspects of the infrared subtraction procedure in presence of QCD and QED-like infrared singularities. Lastly, as a non-trivial example, we have presented the full electroweak NLO corrections to the production of a $W$-boson in association with two 
jets within the GOSAM+MADDIPOLE framework.

\section{Acknowledgements}

We thank Stefan Dittmaier, Thomas Hahn, Wolfgang Hollik, Guido Montagna, Fulvio Piccinini and Sandro Uccirati for useful discussions. N.G. wants to thank the University of Naples and the University of Pavia for kind hospitality while parts of this work were carried out. The work of F.T. was partially supported by the INFN Iniziativa Specifica PhenoLNF. The work of M.C. was partially supported by the INFN Iniziativa Specifica QFTATCOLLIDERS. The work of M.C. and F.T. were partially supported by the Italian Ministry of University and Research under the PRIN project 2010YJ2NYW.

[1] CMS Collaboration Collaboration, S. Chatrchyan et al., Observation of a new boson at a mass of $125 \mathrm{GeV}$ with the CMS experiment at the LHC, Phys.Lett. B716 (2012) 30-61, arXiv:1207.7235].

[2] ATLAS Collaboration Collaboration, G. Aad et al., Observation of a new particle in the search for the Standard Model Higgs boson with the ATLAS detector at the LHC, Phys.Lett. B716 (2012) 1-29, arXiv:1207.7214.

[3] F. Englert and R. Brout, Broken Symmetry and the Mass of Gauge Vector Mesons, Phys.Rev.Lett. 13 (1964) 321-323.

[4] P. W. Higgs, Broken symmetries, massless particles and gauge fields, Phys. Lett. 12 (1964) $132-133$.

[5] P. W. Higgs, Broken Symmetries and the Masses of Gauge Bosons, Phys.Rev.Lett. 13 (1964) $508-509$.

[6] G. Guralnik, C. Hagen, and T. Kibble, Global Conservation Laws and Massless Particles, Phys.Rev.Lett. 13 (1964) 585-587.

[7] T. Kibble, Symmetry breaking in nonAbelian gauge theories, Phys.Rev. 155 (1967) 1554-1561.

[8] G. Corcella, I. G. Knowles, G. Marchesini, S. Moretti, K. Odagiri, P. Richardson, M. H. Seymour, and B. R. Webber, HERWIG 6: An Event generator for hadron emission reactions 
with interfering gluons (including supersymmetric processes), JHEP 01 (2001) 010, hep-ph/0011363.

[9] M. Bahr et al., Herwig ++ Physics and Manual, Eur. Phys. J. C58 (2008) 639-707, arXiv:0803.0883].

[10] T. Sjostrand, S. Mrenna, and P. Z. Skands, PYTHIA 6.4 Physics and Manual, JHEP 05 (2006) 026, hep-ph/0603175.

[11] T. Sjostrand, S. Mrenna, and P. Z. Skands, A Brief Introduction to PYTHIA 8.1, Comput. Phys. Commun. 178 (2008) 852-867, [arXiv:0710.3820].

[12] T. Gleisberg, S. Hoeche, F. Krauss, M. Schonherr, S. Schumann, et al., Event generation with SHERPA 1.1, JHEP 0902 (2009) 007, arXiv:0811.4622].

[13] G. Ossola, C. G. Papadopoulos, and R. Pittau, Reducing full one-loop amplitudes to scalar integrals at the integrand level, Nucl.Phys. B763 (2007) 147-169, hep-ph/0609007.

[14] F. del Aguila and R. Pittau, Recursive numerical calculus of one-loop tensor integrals, JHEP 0407 (2004) 017, hep-ph/0404120.

[15] P. Mastrolia, G. Ossola, T. Reiter, and F. Tramontano, Scattering AMplitudes from Unitarity-based Reduction Algorithm at the Integrand-level, JHEP 1008 (2010) 080, arXiv: 1006.0710 .

[16] Z. Bern, L. J. Dixon, D. C. Dunbar, and D. A. Kosower, One loop n point gauge theory amplitudes, unitarity and collinear limits, Nucl.Phys. B425 (1994) 217-260, hep-ph/9403226.

[17] R. K. Ellis, W. Giele, and Z. Kunszt, A Numerical Unitarity Formalism for Evaluating One-Loop Amplitudes, JHEP 0803 (2008) 003, arXiv:0708.2398].

[18] R. K. Ellis, W. T. Giele, Z. Kunszt, and K. Melnikov, Masses, fermions and generalized D-dimensional unitarity, Nucl.Phys. B822 (2009) 270-282, arXiv:0806.3467.

[19] C. Berger, Z. Bern, L. Dixon, F. Febres Cordero, D. Forde, et al., An Automated Implementation of On-Shell Methods for One-Loop Amplitudes, Phys.Rev. D78 (2008) 036003, arXiv:0803.4180].

[20] S. Badger, B. Biedermann, P. Uwer, and V. Yundin, Numerical evaluation of virtual corrections to multi-jet production in massless QCD, Comput.Phys.Commun. 184 (2013) 1981-1998, [arXiv:1209.0100].

[21] G. Cullen, N. Greiner, G. Heinrich, G. Luisoni, P. Mastrolia, et al., Automated One-Loop 
Calculations with GoSam, Eur.Phys.J. C72 (2012) 1889, [arXiv:1111.2034].

[22] G. Cullen, H. van Deurzen, N. Greiner, G. Heinrich, G. Luisoni, et al., GOSAM-2.0: a tool for automated one-loop calculations within the Standard Model and beyond, Eur.Phys.J. C74 (2014), no. 8 3001, arXiv:1404.7096].

[23] G. Bevilacqua, M. Czakon, M. Garzelli, A. van Hameren, A. Kardos, et al., HELAC-NLO, Comput.Phys.Commun. 184 (2013) 986-997, [arXiv:1110.1499].

[24] V. Hirschi, R. Frederix, S. Frixione, M. V. Garzelli, F. Maltoni, et al., Automation of one-loop QCD corrections, JHEP 1105 (2011) 044, [arXiv:1103.0621].

[25] J. Alwall, M. Herquet, F. Maltoni, O. Mattelaer, and T. Stelzer, MadGraph 5 : Going Beyond, JHEP 1106 (2011) 128, arXiv:1106.0522.

[26] J. Alwall, R. Frederix, S. Frixione, V. Hirschi, F. Maltoni, et al., The automated computation of tree-level and next-to-leading order differential cross sections, and their matching to parton shower simulations, JHEP 1407 (2014) 079, arXiv:1405.0301].

[27] F. Cascioli, P. Maierhofer, and S. Pozzorini, Scattering Amplitudes with Open Loops, Phys.Rev.Lett. 108 (2012) 111601, arXiv:1111.5206.

[28] S. Actis, A. Denner, L. Hofer, A. Scharf, and S. Uccirati, Recursive generation of one-loop amplitudes in the Standard Model, JHEP 1304 (2013) 037, arXiv:1211.6316.

[29] M. L. Mangano, M. Moretti, F. Piccinini, R. Pittau, and A. D. Polosa, ALPGEN, a generator for hard multiparton processes in hadronic collisions, JHEP 0307 (2003) 001, hep-ph/0206293.

[30] S. Catani, F. Krauss, R. Kuhn, and B. Webber, QCD matrix elements + parton showers, JHEP 0111 (2001) 063, hep-ph/0109231.

[31] F. Krauss, Matrix elements and parton showers in hadronic interactions, JHEP 0208 (2002) 015, hep-ph/0205283.

[32] M. L. Mangano, M. Moretti, F. Piccinini, and M. Treccani, Matching matrix elements and shower evolution for top-quark production in hadronic collisions, JHEP 0701 (2007) 013, hep-ph/0611129.

[33] P. Nason, A New method for combining NLO QCD with shower Monte Carlo algorithms, JHEP 0411 (2004) 040, hep-ph/0409146.

[34] S. Frixione, P. Nason, and C. Oleari, Matching NLO QCD computations with Parton Shower 
simulations: the POWHEG method, JHEP 0711 (2007) 070, arXiv:0709.2092.

[35] S. Alioli, P. Nason, C. Oleari, and E. Re, A general framework for implementing NLO calculations in shower Monte Carlo programs: the POWHEG BOX, JHEP 1006 (2010) 043, [arXiv:1002.2581].

[36] S. Frixione and B. R. Webber, Matching NLO QCD computations and parton shower simulations, JHEP 0206 (2002) 029, hep-ph/0204244.

[37] R. Frederix and S. Frixione, Merging meets matching in MC@NLO, JHEP 1212 (2012) 061, [arXiv:1209.6215].

[38] T. Gehrmann, S. Hoche, F. Krauss, M. Schonherr, and F. Siegert, NLO QCD matrix elements + parton showers in $e^{+} e^{-} \rightarrow$ hadrons, JHEP 1301 (2013) 144, arXiv:1207.5031].

[39] S. Hoeche, F. Krauss, M. Schonherr, and F. Siegert, QCD matrix elements + parton showers: The NLO case, JHEP 1304 (2013) 027, arXiv:1207.5030].

[40] K. Hamilton, P. Nason, and G. Zanderighi, MINLO: Multi-Scale Improved NLO, JHEP 10 (2012) 155, arXiv:1206.3572.

[41] K. Hamilton, P. Nason, C. Oleari, and G. Zanderighi, Merging $H / W / Z+O$ and 1 jet at $N L O$ with no merging scale: a path to parton shower + NNLO matching, JHEP 1305 (2013) 082, arXiv:1212.4504].

[42] L. Lonnblad and S. Prestel, Merging Multi-leg NLO Matrix Elements with Parton Showers, JHEP 1303 (2013) 166, arXiv:1211.7278.

[43] P. Golonka and Z. Was, PHOTOS Monte Carlo: A Precision tool for QED corrections in Z and $W$ decays, Eur. Phys. J. C45 (2006) 97-107, hep-ph/0506026.

[44] R. Frederix, T. Gehrmann, and N. Greiner, Automation of the Dipole Subtraction Method in MadGraph/MadEvent, JHEP 0809 (2008) 122, arXiv:0808.2128].

[45] R. Frederix, T. Gehrmann, and N. Greiner, Integrated dipoles with MadDipole in the MadGraph framework, JHEP 1006 (2010) 086, arXiv:1004.2905].

[46] T. Gehrmann and N. Greiner, Photon Radiation with MadDipole, JHEP 1012 (2010) 050, arXiv:1011.0321].

[47] C. Carloni Calame, G. Montagna, O. Nicrosini, and A. Vicini, Precision electroweak calculation of the charged current Drell-Yan process, JHEP 0612 (2006) 016, hep-ph/0609170.

[48] C. Carloni Calame, G. Montagna, O. Nicrosini, and A. Vicini, Precision electroweak calculation 
of the production of a high transverse-momentum lepton pair at hadron colliders, JHEP 0710 (2007) 109, arXiv:0710.1722.

[49] S. Dittmaier and. Kramer, Michael, Electroweak radiative corrections to $W$ boson production at hadron colliders, Phys.Rev. D65 (2002) 073007, hep-ph/0109062.

[50] S. Brensing, S. Dittmaier, . Kramer, Michael, and A. Muck, Radiative corrections to $W^{-}$boson hadroproduction: Higher-order electroweak and supersymmetric effects, Phys.Rev. D77 (2008) 073006, arXiv:0710.3309].

[51] S. Dittmaier and M. Huber, Radiative corrections to the neutral-current Drell-Yan process in the Standard Model and its minimal supersymmetric extension, JHEP 1001 (2010) 060, [arXiv:0911.2329].

[52] A. Arbuzov, D. Bardin, S. Bondarenko, P. Christova, L. Kalinovskaya, et al., One-loop corrections to the Drell-Yan process in SANC. I. The Charged current case, Eur.Phys.J. C46 (2006) 407-412, hep-ph/0506110.

[53] A. Arbuzov, D. Bardin, S. Bondarenko, P. Christova, L. Kalinovskaya, et al., One-loop corrections to the Drell-Yan process in SANC. (II). The Neutral current case, Eur.Phys.J. C54 (2008) 451-460, arXiv:0711.0625].

[54] U. Baur and D. Wackeroth, Electroweak radiative corrections to $p \bar{p} \rightarrow W^{ \pm} \rightarrow \ell^{ \pm} \nu$ beyond the pole approximation, Phys.Rev. D70 (2004) 073015, hep-ph/0405191.

[55] W. Placzek and S. Jadach, Multiphoton radiation in leptonic $W$ boson decays, Eur.Phys.J. C29 (2003) 325-339, hep-ph/0302065.

[56] D. Bardin, S. Bondarenko, S. Jadach, L. Kalinovskaya, and W. Placzek, Implementation of SANC EW corrections in WINHAC Monte Carlo generator, Acta Phys.Polon. B40 (2009) 75-92, arXiv:0806.3822.

[57] U. Baur, O. Brein, W. Hollik, C. Schappacher, and D. Wackeroth, Electroweak radiative corrections to neutral current Drell-Yan processes at hadron colliders, Phys.Rev. D65 (2002) 033007, hep-ph/0108274.

[58] Y. Li and F. Petriello, Combining QCD and electroweak corrections to dilepton production in FEWZ, Phys.Rev. D86 (2012) 094034, [arXiv:1208.5967].

[59] L. Barze, G. Montagna, P. Nason, O. Nicrosini, and F. Piccinini, Implementation of electroweak corrections in the POWHEG BOX: single W production, JHEP 1204 (2012) 037, 
arXiv:1202.0465].

[60] C. Bernaciak and D. Wackeroth, Combining NLO QCD and Electroweak Radiative Corrections to $W$ boson Production at Hadron Colliders in the POWHEG Framework, Phys. Rev. D85 (2012) 093003, arXiv:1201.4804.

[61] L. Barze, G. Montagna, P. Nason, O. Nicrosini, F. Piccinini, et al., Neutral current Drell-Yan with combined QCD and electroweak corrections in the POWHEG BOX, Eur.Phys.J. C73 (2013), no. 6 2474, arXiv:1302.4606].

[62] J. Butterworth, G. Dissertori, S. Dittmaier, D. de Florian, N. Glover, et al., Les Houches 2013: Physics at TeV Colliders: Standard Model Working Group Report, arXiv:1405.1067.

[63] S. Boselli, C. M. C. Calame, G. Montagna, O. Nicrosini, and F. Piccinini, Higgs boson decay into four leptons at NLOPS electroweak accuracy, JHEP 1506 (2015) 023, [arXiv:1503.0739].

[64] S. Alekhin, J. Blumlein, and S. Moch, The ABM parton distributions tuned to LHC data, Phys. Rev. D89 (2014), no. 5 054028, [arXiv:1310.3059].

[65] NNPDF Collaboration, R. D. Ball et al., Parton distributions for the LHC Run II, JHEP 04 (2015) 040, arXiv:1410.8849.

[66] L. A. Harland-Lang, A. D. Martin, P. Motylinski, and R. S. Thorne, Parton distributions in the LHC era: MMHT 2014 PDFs, Eur. Phys. J. C75 (2015), no. 5 204, arXiv:1412.3989.

[67] S. Dulat, T. J. Hou, J. Gao, M. Guzzi, J. Huston, P. Nadolsky, J. Pumplin, C. Schmidt, D. Stump, and C. P. Yuan, The CT14 Global Analysis of Quantum Chromodynamics, arXiv: 1506.0744

[68] R. Boughezal, Y. Li, and F. Petriello, Disentangling radiative corrections using the high-mass Drell-Yan process at the LHC, Phys. Rev. D89 (2014), no. 3 034030, [arXiv:1312.3972].

[69] A. D. Martin, R. G. Roberts, W. J. Stirling, and R. S. Thorne, Parton distributions incorporating QED contributions, Eur. Phys. J. C39 (2005) 155-161, hep-ph/0411040.

[70] NNPDF Collaboration, R. D. Ball, V. Bertone, S. Carrazza, L. Del Debbio, S. Forte, A. Guffanti, N. P. Hartland, and J. Rojo, Parton distributions with QED corrections, Nucl. Phys. B877 (2013) 290-320, arXiv:1308.0598.

[71] C. Schmidt, J. Pumplin, D. Stump, and C. P. Yuan, CT14QED PDFs from Isolated Photon Production in Deep Inelastic Scattering, arXiv:1509.0290.

[72] V. Bertone, S. Carrazza, and J. Rojo, APFEL: A PDF Evolution Library with QED 
corrections, Comput. Phys. Commun. 185 (2014) 1647-1668, arXiv:1310.1394].

[73] S. Carrazza, A. Ferrara, D. Palazzo, and J. Rojo, APFEL Web: a web-based application for the graphical visualization of parton distribution functions, J. Phys. G42 (2015), no. 5 057001, [arXiv:1410.5456].

[74] M. Ciafaloni, P. Ciafaloni, and D. Comelli, Towards collinear evolution equations in electroweak theory, Phys. Rev. Lett. 88 (2002) 102001, hep-ph/0111109.

[75] P. Ciafaloni and D. Comelli, Electroweak evolution equations, JHEP 11 (2005) 022, hep-ph/0505047.

[76] ATLAS Collaboration, G. Aad et al., Measurements of the $W$ production cross sections in association with jets with the ATLAS detector, Eur.Phys.J. C75 (2015), no. 2 82, [arXiv:1409.8639].

[77] CMS Collaboration, V. Khachatryan et al., Differential cross section measurements for the production of a $W$ boson in association with jets in proton-proton collisions at $\sqrt{s}=7 \mathrm{TeV}$, Phys.Lett. B741 (2015) 12-37, arXiv:1406.7533.

[78] ATLAS Collaboration, G. Aad et al., Measurement of the production cross section of jets in association with a $Z$ boson in pp collisions at $\sqrt{s}=7$ TeV with the ATLAS detector, JHEP 1307 (2013) 032, arXiv:1304.7098.

[79] CMS Collaboration, V. Khachatryan et al., Measurements of jet multiplicity and differential production cross sections of $Z+$ jets events in proton-proton collisions at $\sqrt{s}=7 \mathrm{TeV}$, Phys.Rev. D91 (2015), no. 5 052008, [arXiv:1408.3104].

[80] ATLAS Collaboration, G. Aad et al., A measurement of the ratio of the production cross sections for $W$ and $Z$ bosons in association with jets with the ATLAS detector, Eur.Phys.J. C74 (2014), no. 12 3168, arXiv:1408.6510].

[81] CMS Collaboration, V. Khachatryan et al., Comparison of the Z/gamma*+jets to gamma+jets cross sections in pp collisions at sqrt(s)=8 TeV, arXiv:1505.0652,

[82] CMS Collaboration, S. Chatrchyan et al., Search for new physics in the multijet and missing transverse momentum final state in proton-proton collisions at $\sqrt{s}=8 \mathrm{TeV}$, JHEP 1406 (2014) 055, arXiv:1402.4770.

[83] ATLAS Collaboration, G. Aad et al., Search for new phenomena in final states with large jet multiplicities and missing transverse momentum at $\sqrt{s}=8$ TeV proton-proton collisions using 
the ATLAS experiment, JHEP 1310 (2013) 130, arXiv:1308.1841.

[84] CMS Collaboration, V. Khachatryan et al., Search for stealth supersymmetry in events with jets, either photons or leptons, and low missing transverse momentum in pp collisions at 8 TeV, Phys.Lett. B743 (2015) 503-525, arXiv:1411.7255].

[85] ATLAS Collaboration, G. Aad et al., Search for squarks and gluinos in events with isolated leptons, jets and missing transverse momentum at $\sqrt{s}=8 \mathrm{TeV}$ with the ATLAS detector, JHEP 1504 (2015) 116, arXiv:1501.0355.

[86] V. Sudakov, Vertex parts at very high-energies in quantum electrodynamics, Sov.Phys.JETP 3 (1956) 65-71.

[87] M. Beccaria, G. Montagna, F. Piccinini, F. Renard, and C. Verzegnassi, Rising bosonic electroweak virtual effects at high-energy e+ e- colliders, Phys.Rev. D58 (1998) 093014, hep-ph/9805250.

[88] P. Ciafaloni and D. Comelli, Sudakov enhancement of electroweak corrections, Phys.Lett. B446 (1999) 278-284, hep-ph/9809321.

[89] M. Beccaria, P. Ciafaloni, D. Comelli, F. Renard, and C. Verzegnassi, Logarithmic expansion of electroweak corrections to four-fermion processes in the TeV region, Phys.Rev. D61 (2000) 073005, hep-ph/9906319.

[90] M. Ciafaloni, P. Ciafaloni, and D. Comelli, Bloch-Nordsieck violating electroweak corrections to inclusive TeV scale hard processes, Phys.Rev.Lett. 84 (2000) 4810-4813, hep-ph/0001142.

[91] M. Ciafaloni, P. Ciafaloni, and D. Comelli, Electroweak Bloch-Nordsieck violation at the TeV scale: 'Strong' weak interactions?, Nucl.Phys. B589 (2000) 359-380, hep-ph/0004071.

[92] M. Ciafaloni, P. Ciafaloni, and D. Comelli, Bloch-Nordsieck violation in spontaneously broken Abelian theories, Phys.Rev.Lett. 87 (2001) 211802, hep-ph/0103315.

[93] V. S. Fadin, L. Lipatov, A. D. Martin, and M. Melles, Resummation of double logarithms in electroweak high-energy processes, Phys.Rev. D61 (2000) 094002, hep-ph/9910338.

[94] W. Stirling and E. Vryonidou, Electroweak corrections and Bloch-Nordsieck violations in 2-to-2 processes at the LHC, JHEP 1304 (2013) 155, [arXiv:1212.6537.

[95] A. Denner and S. Pozzorini, One loop leading logarithms in electroweak radiative corrections. 1. Results, Eur.Phys.J. C18 (2001) 461-480, hep-ph/0010201.

[96] A. Denner and S. Pozzorini, One loop leading logarithms in electroweak radiative corrections. 
2. Factorization of collinear singularities, Eur.Phys.J. C21 (2001) 63-79, hep-ph/0104127.

[97] M. Chiesa, G. Montagna, L. Barzè, M. Moretti, O. Nicrosini, et al., Electroweak Sudakov Corrections to New Physics Searches at the LHC, Phys.Rev.Lett. 111 (2013), no. 12 121801, [arXiv:1305.6837].

[98] K. Mishra, T. Becher, L. Barze, M. Chiesa, S. Dittmaier, et al., Electroweak Corrections at High Energies, arXiv:1308.1430,

[99] J. Campbell, K. Hatakeyama, J. Huston, F. Petriello, J. R. Andersen, et al., Working Group Report: Quantum Chromodynamics, arXiv:1310.5189.

[100] E. Maina, S. Moretti, and D. A. Ross, One loop weak corrections to gamma / Z hadroproduction at finite transverse momentum, Phys.Lett. B593 (2004) 143-150, hep-ph/0403050.

[101] J. H. Kuhn, A. Kulesza, S. Pozzorini, and M. Schulze, Electroweak corrections to hadronic photon production at large transverse momenta, JHEP 0603 (2006) 059, hep-ph/0508253.

[102] J. H. Kuhn, A. Kulesza, S. Pozzorini, and M. Schulze, One-loop weak corrections to hadronic production of $Z$ bosons at large transverse momenta, Nucl.Phys. B727 (2005) 368-394, hep-ph/0507178.

[103] J. H. Kuhn, A. Kulesza, S. Pozzorini, and M. Schulze, Electroweak corrections to hadronic production of $W$ bosons at large transverse momenta, Nucl.Phys. B797 (2008) 27-77, arXiv:0708.0476.

[104] A. Denner, S. Dittmaier, T. Kasprzik, and A. Muck, Electroweak corrections to $W+$ jet hadroproduction including leptonic W-boson decays, JHEP 0908 (2009) 075, arXiv:0906.1656].

[105] A. Denner, S. Dittmaier, T. Kasprzik, and A. Muck, Electroweak corrections to dilepton + jet production at hadron colliders, JHEP 1106 (2011) 069, [arXiv:1103.0914].

[106] A. Denner, S. Dittmaier, T. Kasprzik, and A. Mück, Electroweak corrections to monojet production at the LHC, Eur.Phys.J. C73 (2013), no. 2 2297, arXiv:1211.5078.

[107] M. Beccaria, G. Macorini, F. Renard, and C. Verzegnassi, Single top production in the t-channel at LHC: A Realistic test of electroweak models, Phys.Rev. D74 (2006) 013008, hep-ph/0605108.

[108] M. Beccaria, C. Carloni Calame, G. Macorini, E. Mirabella, F. Piccinini, et al., A Complete 
one-loop calculation of electroweak supersymmetric effects in $t$-channel single top production at CERN LHC, Phys.Rev. D77 (2008) 113018, arXiv:0802.1994.

[109] D. Bardin, S. Bondarenko, L. Kalinovskaya, V. Kolesnikov, and W. von Schlippe, Electroweak Radiative Corrections to Single-top Production, Eur.Phys.J. C71 (2011) 1533, arXiv: 1008.1859].

[110] W. Beenakker, A. Denner, W. Hollik, R. Mertig, T. Sack, et al., Electroweak one loop contributions to top pair production in hadron colliders, Nucl.Phys. B411 (1994) 343-380.

[111] J. H. Kuhn, A. Scharf, and P. Uwer, Electroweak corrections to top-quark pair production in quark-antiquark annihilation, Eur.Phys.J. C45 (2006) 139-150, hep-ph/0508092.

[112] S. Moretti, M. Nolten, and D. Ross, Weak corrections to gluon-induced top-antitop hadro-production, Phys.Lett. B639 (2006) 513-519, hep-ph/0603083.

[113] W. Bernreuther, M. Fuecker, and Z.-G. Si, Weak interaction corrections to hadronic top quark pair production, Phys.Rev. D74 (2006) 113005, hep-ph/0610334.

[114] J. H. Kuhn, A. Scharf, and P. Uwer, Electroweak effects in top-quark pair production at hadron colliders, Eur.Phys.J. C51 (2007) 37-53, hep-ph/0610335.

[115] W. Hollik and M. Kollar, NLO QED contributions to top-pair production at hadron collider, Phys.Rev. D77 (2008) 014008, arXiv:0708.1697.

[116] W. Bernreuther, M. Fucker, and Z.-G. Si, Weak interaction corrections to hadronic top quark pair production: Contributions from quark-gluon and b anti-b induced reactions, Phys.Rev. D78 (2008) 017503, [arXiv:0804.1237].

[117] S. Moretti, M. Nolten, and D. Ross, Weak corrections and high E(T) jets at Tevatron, Phys.Rev. D74 (2006) 097301, hep-ph/0503152].

[118] S. Moretti, M. Nolten, and D. Ross, Weak corrections to four-parton processes, Nucl.Phys. B759 (2006) 50-82, hep-ph/0606201.

[119] S. Dittmaier, A. Huss, and C. Speckner, Weak radiative corrections to dijet production at hadron colliders, JHEP 1211 (2012) 095, arXiv:1210.0438.

[120] A. Denner, S. Dittmaier, S. Kallweit, and A. Muck, Electroweak corrections to Higgs-strahlung off $W / Z$ bosons at the Tevatron and the LHC with HAWK, JHEP 1203 (2012) 075, [arXiv:1112.5142].

[121] M. Ciccolini, S. Dittmaier, and M. Kramer, Electroweak radiative corrections to associated WH 
and ZH production at hadron colliders, Phys.Rev. D68 (2003) 073003, hep-ph/0306234.

[122] M. Ciccolini, A. Denner, and S. Dittmaier, Electroweak and QCD corrections to Higgs production via vector-boson fusion at the LHC, Phys.Rev. D77 (2008) 013002, arXiv:0710.4749].

[123] M. Ciccolini, A. Denner, and S. Dittmaier, Strong and electroweak corrections to the production of Higgs + 2jets via weak interactions at the LHC, Phys.Rev.Lett. 99 (2007) 161803, arXiv:0707.0381].

[124] E. Accomando, A. Denner, and C. Meier, Electroweak corrections to $W \gamma$ and $Z \gamma$ production at the LHC, Eur.Phys.J. C47 (2006) 125-146, hep-ph/0509234.

[125] A. Bierweiler, T. Kasprzik, J. H. Kuhn, and S. Uccirati, Electroweak corrections to W-boson pair production at the LHC, JHEP 1211 (2012) 093, [arXiv:1208.3147].

[126] M. Billoni, S. Dittmaier, B. Jager, and C. Speckner, Next-to-leading order electroweak corrections to $p p \rightarrow W+W-\rightarrow 4$ leptons at the LHC in double-pole approximation, JHEP 1312 (2013) 043, arXiv:1310.1564.

[127] A. Bierweiler, T. Kasprzik, and J. H. Kuhn, Vector-boson pair production at the LHC to $\mathcal{O}\left(\alpha^{3}\right)$ accuracy, JHEP 1312 (2013) 071, [arXiv:1305.5402].

[128] S. Gieseke, T. Kasprzik, and J. H. Kuhn, Vector-boson pair production and electroweak corrections in HERWIG++, Eur.Phys.J. C74 (2014), no. 8 2988, arXiv:1401.3964.

[129] J. Baglio, L. D. Ninh, and M. M. Weber, Massive gauge boson pair production at the LHC: a next-to-leading order story, Phys.Rev. D88 (2013) 113005, [arXiv:1307.4331].

[130] L. Wei-Hua, Z. Ren-You, M. Wen-Gan, G. Lei, L. Xiao-Zhou, and Z. Yu, NLO QCD and EW corrections to $W W+$ jet production with leptonic $W$-boson decays at LHC, arXiv:1507.0733.

[131] D. T. Nhung, L. D. Ninh, and M. M. Weber, NLO corrections to WWZ production at the LHC, JHEP 1312 (2013) 096, arXiv:1307.7403.

[132] S. Yong-Bai, Z. Ren-You, M. Wen-Gan, L. Xiao-Zhou, Z. Yu, and G. Lei, $N L O Q C D+N L O$ $E W$ corrections to $W Z Z$ productions with leptonic decays at the $L H C$, arXiv:1507.0369.

[133] A. Bredenstein, A. Denner, S. Dittmaier, and M. Weber, Radiative corrections to the semileptonic and hadronic Higgs-boson decays $H \rightarrow W W / Z Z \rightarrow 4$ fermions, JHEP 0702 (2007) 080, hep-ph/0611234.

[134] A. Denner, S. Dittmaier, M. Hecht, and C. Pasold, NLO QCD and electroweak corrections to 
$W+\gamma$ production with leptonic $W$-boson decays, JHEP 1504 (2015) 018, arXiv:1412.7421.

[135] S. Frixione, V. Hirschi, D. Pagani, H. Shao, and M. Zaro, Weak corrections to Higgs hadroproduction in association with a top-quark pair, JHEP 1409 (2014) 065, [arXiv:1407.0823].

[136] Y. Zhang, W.-G. Ma, R.-Y. Zhang, C. Chen, and L. Guo, QCD NLO and EW NLO corrections to $t \bar{t} H$ production with top quark decays at hadron collider, Phys. Lett. B738 (2014) 1-5, arXiv:1407.1110].

[137] A. Denner, L. Hofer, A. Scharf, and S. Uccirati, Electroweak corrections to lepton pair production in association with two hard jets at the LHC, JHEP 1501 (2015) 094, [arXiv:1411.0916].

[138] S. Kallweit, J. M. Lindert, P. Maierhöfer, S. Pozzorini, and M. Schönherr, NLO electroweak automation and precise predictions for $W+$ multijet production at the $L H C$, arXiv:1412.5157.

[139] S. Frixione, V. Hirschi, D. Pagani, H. S. Shao, and M. Zaro, Electroweak and QCD corrections to top-pair hadroproduction in association with heavy bosons, arXiv:1504.0344.

[140] J.-y. Chiu, R. Kelley, and A. V. Manohar, Electroweak Corrections using Effective Field Theory: Applications to the LHC, Phys.Rev. D78 (2008) 073006, [arXiv:0806.1240].

[141] E. Accomando, A. Denner, and A. Kaiser, Logarithmic electroweak corrections to gauge-boson pair production at the LHC, Nucl.Phys. B706 (2005) 325-371, hep-ph/0409247.

[142] E. Accomando and A. Kaiser, Electroweak corrections and anomalous triple gauge-boson couplings in $W^{+} W^{-}$and $W^{ \pm} Z$ production at the LHC, Phys.Rev. D73 (2006) 093006, hep-ph/0511088.

[143] A. Fuhrer, A. V. Manohar, J.-y. Chiu, and R. Kelley, Radiative Corrections to Longitudinal and Transverse Gauge Boson and Higgs Production, Phys.Rev. D81 (2010) 093005, arXiv:1003.0025.

[144] T. Becher and X. Garcia i Tormo, Electroweak Sudakov effects in W, Z and /gamma production at large transverse momentum, Phys.Rev. D88 (2013), no. 1 013009, arXiv:1305.4202].

[145] A. V. Manohar and M. Trott, Electroweak Sudakov Corrections and the Top Quark Forward-Backward Asymmetry, Phys.Lett. B711 (2012) 313-316, [arXiv:1201.3926].

[146] A. Fuhrer, A. V. Manohar, and W. J. Waalewijn, Electroweak radiative Corrections to Higgs 
Production via Vector Boson Fusion using Soft-Collinear Effective Theory, Phys.Rev. D84 (2011) 013007, arXiv:1011.1505.

[147] F. Siringo and G. Buccheri, Electroweak Radiative Corrections to Higgs Production via Vector Boson Fusion using SCET: Numerical Results, Phys.Rev. D86 (2012) 053013, arXiv:1207.1906].

[148] J. M. Campbell and R. K. Ellis, MCFM for the Tevatron and the LHC, Nucl. Phys. Proc. Suppl. 205-206 (2010) 10-15, arXiv:1007.3492.

[149] J. M. Campbell, D. Wackeroth, and J. Zhou, Electroweak Corrections at the LHC with MCFM, in 23rd International Workshop on Deep-Inelastic Scattering and Related Subjects (DIS 2015) Dallas, Texas, United States, April 27-May 1, 2015, 2015. arXiv:1508.0624.

[150] A. Denner, S. Dittmaier, and L. Hofer, COLLIER - A fortran-library for one-loop integrals, PoS LL2014 (2014) 071, arXiv:1407.0087.

[151] A. Denner and S. Dittmaier, Reduction of one loop tensor five point integrals, Nucl.Phys. B658 (2003) 175-202, hep-ph/0212259.

[152] A. Denner and S. Dittmaier, Reduction schemes for one-loop tensor integrals, Nucl.Phys. B734 (2006) 62-115, hep-ph/0509141.

[153] S. Catani and M. Seymour, A General algorithm for calculating jet cross-sections in NLO QCD, Nucl.Phys. B485 (1997) 291-419, hep-ph/9605323.

[154] S. Catani, S. Dittmaier, M. H. Seymour, and Z. Trocsanyi, The Dipole formalism for next-to-leading order QCD calculations with massive partons, Nucl.Phys. B627 (2002) 189-265, hep-ph/0201036.

[155] S. Frixione, Z. Kunszt, and A. Signer, Three jet cross-sections to next-to-leading order, Nucl.Phys. B467 (1996) 399-442, hep-ph/9512328.

[156] S. Frixione, A General approach to jet cross-sections in QCD, Nucl.Phys. B507 (1997) 295-314, hep-ph/9706545.

[157] R. Frederix, S. Frixione, F. Maltoni, and T. Stelzer, Automation of next-to-leading order computations in QCD: The FKS subtraction, JHEP 0910 (2009) 003, arXiv:0908.4272.

[158] R. K. Ellis, D. Ross, and A. Terrano, The Perturbative Calculation of Jet Structure in e+ eAnnihilation, Nucl.Phys. B178 (1981) 421.

[159] G. 't Hooft and M. Veltman, Regularization and Renormalization of Gauge Fields, Nucl.Phys. 
B44 (1972) 189-213.

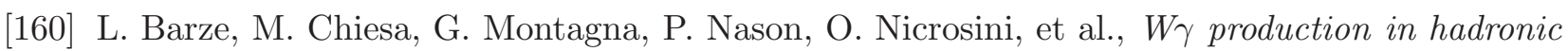
collisions using the POWHEG+MiNLO method, JHEP 1412 (2014) 039, arXiv:1408.5766.

[161] D. Akyeampong and R. Delbourgo, Dimensional regularization, abnormal amplitudes and anomalies, Nuovo Cim. A17 (1973) 578-586.

[162] D. Akyeampong and R. Delbourgo, Dimensional regularization and PCAC, Nuovo Cim. A18 (1973) 94-104.

[163] P. Breitenlohner and D. Maison, Dimensional Renormalization and the Action Principle, Commun.Math.Phys. 52 (1977) 11-38.

[164] J. Korner, N. Nasrallah, and K. Schilcher, Evaluation of the Flavor Changing Vertex $b \rightarrow s H$ Using the Breitenlohner-maison-'t Hooft-Veltman $\gamma(5)$ Scheme, Phys.Rev. D41 (1990) 888.

[165] D. Kreimer, The Role of gamma(5) in dimensional regularization, hep-ph/9401354.

[166] J. Korner, D. Kreimer, and K. Schilcher, A Practicable gamma(5) scheme in dimensional regularization, Z.Phys. C54 (1992) 503-512.

[167] M. S. Chanowitz, M. Furman, and I. Hinchliffe, The Axial Current in Dimensional Regularization, Nucl. Phys. B159 (1979) 225.

[168] T. Hahn and M. Perez-Victoria, Automatized one loop calculations in four-dimensions and D-dimensions, Comput.Phys.Commun. 118 (1999) 153-165, hep-ph/9807565.

[169] C. Schubert, The Yukawa Model as an Example for Dimensional Renormalization With $\gamma$ (5), Nucl.Phys. B323 (1989) 478.

[170] R. Ferrari, A. Le Yaouanc, L. Oliver, and J. Raynal, Gauge invariance and dimensional regularization with gamma(5) in flavor changing neutral processes, Phys.Rev. D52 (1995) 3036-3047.

[171] A. Denner, Techniques for calculation of electroweak radiative corrections at the one loop level and results for W physics at LEP-200, Fortsch.Phys. 41 (1993) 307-420, [arXiv:0709.1075].

[172] C. Bollini and J. Giambiagi, Dimensional Renormalization: The Number of Dimensions as a Regularizing Parameter, Nuovo Cim. 12 (1972), no. 1 20-26.

[173] J. Ashmore, A Method of Gauge Invariant Regularization, Lett.Nuovo Cim. 4 (1972) 289-290.

[174] G. Cicuta and E. Montaldi, Analytic renormalization via continuous space dimension, Lett.Nuovo Cim. 4 (1972) 329-332. 
[175] Z. Bern, L. J. Dixon, and D. A. Kosower, Dimensionally regulated one loop integrals, Phys.Lett. B302 (1993) 299-308, hep-ph/9212308.

[176] Z. Kunszt, A. Signer, and Z. Trocsanyi, One loop helicity amplitudes for all $2 \rightarrow 2$ processes in QCD and N=1 supersymmetric Yang-Mills theory, Nucl.Phys. B411 (1994) 397-442, hep-ph/9305239.

[177] W. Siegel, Supersymmetric Dimensional Regularization via Dimensional Reduction, Phys.Lett. B84 (1979) 193.

[178] W. Siegel, Inconsistency of Supersymmetric Dimensional Regularization, Phys.Lett. B94 (1980) 37.

[179] I. Jack, D. Jones, and K. Roberts, Equivalence of dimensional reduction and dimensional regularization, Z.Phys. C63 (1994) 151-160, hep-ph/9401349.

[180] D. Stockinger, Regularization by dimensional reduction: consistency, quantum action principle, and supersymmetry, JHEP 0503 (2005) 076, hep-ph/0503129.

[181] S. Catani, M. Seymour, and Z. Trocsanyi, Regularization scheme independence and unitarity in QCD cross-sections, Phys.Rev. D55 (1997) 6819-6829, hep-ph/9610553.

[182] D. A. Ross and J. C. Taylor, RENORMALIZATION OF A UNIFIED THEORY OF WEAK AND ELECTROMAGNETIC INTERACTIONS, Nucl. Phys. B51 (1973) 125-144. [Erratum: Nucl. Phys.B58,643(1973)].

[183] A. Sirlin, Radiative Corrections in the SU(2)-L $x$ U(1) Theory: A Simple Renormalization Framework, Phys.Rev. D22 (1980) 971-981.

[184] W. Marciano and A. Sirlin, Radiative Corrections to Neutrino Induced Neutral Current Phenomena in the SU(2)-L x U(1) Theory, Phys.Rev. D22 (1980) 2695.

[185] A. Sirlin and W. Marciano, Radiative Corrections to Muon-neutrino $N \rightarrow m u-X$ and their

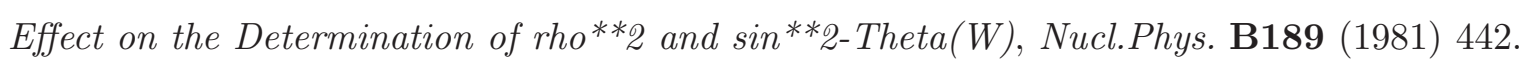

[186] S. Sakakibara, Radiative Corrections to the Neutral Current Interactions in the Weinberg-Salam Model, Phys. Rev. D24 (1981) 1149.

[187] J. Fleischer and F. Jegerlehner, Radiative Corrections to Higgs Decays in the Extended Weinberg-Salam Model, Phys. Rev. D23 (1981) 2001-2026.

[188] D. Yu. Bardin, P. K. Khristova, and O. M. Fedorenko, On the Lowest Order Electroweak Corrections to Spin 1/2 Fermion Scattering. 1. The One Loop Diagrammar, Nucl. Phys. B175 
(1980) 435.

[189] D. Yu. Bardin, P. K. Khristova, and O. M. Fedorenko, On the Lowest Order Electroweak Corrections to Spin 1/2 Fermion Scattering. 2. The One Loop Amplitudes, Nucl. Phys. B197 (1982) 1.

[190] K. I. Aoki, Z. Hioki, M. Konuma, R. Kawabe, and T. Muta, Electroweak Theory. Framework of On-Shell Renormalization and Study of Higher Order Effects, Prog. Theor. Phys. Suppl. 73 (1982) 1-225.

[191] M. Bohm, H. Spiesberger, and W. Hollik, On the One Loop Renormalization of the Electroweak Standard Model and Its Application to Leptonic Processes, Fortsch. Phys. 34 (1986) 687-751.

[192] A. Denner, S. Dittmaier, M. Roth, and D. Wackeroth, Predictions for all processes e+ e- $\rightarrow 4$ fermions + gamma, Nucl.Phys. B560 (1999) 33-65, hep-ph/9904472.

[193] A. Denner, S. Dittmaier, M. Roth, and L. Wieders, Electroweak corrections to charged-current $e+e-\rightarrow 4$ fermion processes: Technical details and further results, Nucl.Phys. B724 (2005) 247-294, hep-ph/0505042.

[194] A. Denner and S. Dittmaier, The Complex-mass scheme for perturbative calculations with unstable particles, Nucl.Phys.Proc.Suppl. 160 (2006) 22-26, hep-ph/0605312.

[195] S. Catani, S. Dittmaier, and Z. Trocsanyi, One loop singular behavior of QCD and SUSY QCD amplitudes with massive partons, Phys.Lett. B500 (2001) 149-160, hep-ph/0011222.

[196] S. Dittmaier, A General approach to photon radiation off fermions, Nucl.Phys. B565 (2000) 69-122, hep-ph/9904440.

[197] S. Dittmaier, A. Kabelschacht, and T. Kasprzik, Polarized QED splittings of massive fermions and dipole subtraction for non-collinear-safe observables, Nucl.Phys. B800 (2008) 146-189, arXiv:0802.1405].

[198] G. 't Hooft and M. Veltman, Scalar One Loop Integrals, Nucl.Phys. B153 (1979) 365-401.

[199] G. Passarino and M. Veltman, One Loop Corrections for e+e-Annihilation Into mu+ mu- in the Weinberg Model, Nucl.Phys. B160 (1979) 151.

[200] D. Y. Bardin and G. Passarino, The standard model in the making: Precision study of the electroweak interactions, .

[201] A. Bredenstein, A. Denner, S. Dittmaier, and S. Pozzorini, NLO QCD corrections to $t$ anti-t $b$ anti-b production at the LHC: 1. Quark-antiquark annihilation, JHEP 0808 (2008) 108, 
arXiv:0807.1248].

[202] T. Kinoshita, Mass singularities of Feynman amplitudes, J.Math.Phys. 3 (1962) 650-677.

[203] T. Lee and M. Nauenberg, Degenerate Systems and Mass Singularities, Phys.Rev. 133 (1964) B1549-B1562.

[204] P. Ciafaloni and D. Comelli, The Importance of weak bosons emission at LHC, JHEP 0609 (2006) 055, hep-ph/0604070.

[205] P. Ciafaloni, D. Comelli, and A. Vergine, Sudakov electroweak effects in transversely polarized beams, JHEP 07 (2004) 039, hep-ph/0311260.

[206] M. Ciafaloni, P. Ciafaloni, and D. Comelli, Enhanced electroweak corrections to inclusive boson fusion processes at the TeV scale, Nucl. Phys. B613 (2001) 382-406, hep-ph/0103316.

[207] G. Bell, J. H. Kuhn, and J. Rittinger, Electroweak Sudakov Logarithms and Real Gauge-Boson Radiation in the TeV Region, Eur. Phys. J. C70 (2010) 659-671, [arXiv:1004.4117.

[208] A. Manohar, B. Shotwell, C. Bauer, and S. Turczyk, Non-cancellation of electroweak logarithms in high-energy scattering, Phys. Lett. B740 (2015) 179-187, [arXiv:1409.1918.

[209] U. Baur, Weak Boson Emission in Hadron Collider Processes, Phys.Rev. D75 (2007) 013005, hep-ph/0611241.

[210] Z. Bern, G. Diana, L. Dixon, F. Febres Cordero, S. Höche, et al., Missing Energy and Jets for Supersymmetry Searches, Phys.Rev. D87 (2013), no. 3 034026, arXiv:1206.6064.

[211] J. R. Christiansen and T. Sjostrand, Weak Gauge Boson Radiation in Parton Showers, JHEP 04 (2014) 115, [arXiv:1401.5238].

[212] F. Krauss, P. Petrov, M. Schoenherr, and M. Spannowsky, Measuring collinear W emissions inside jets, Phys. Rev. D89 (2014), no. 11 114006, arXiv:1403.4788.

[213] E. N. Glover and A. Morgan, Measuring the photon fragmentation function at LEP, Z.Phys. C62 (1994) 311-322.

[214] S. Frixione, Isolated photons in perturbative QCD, Phys.Lett. B429 (1998) 369-374, hep-ph/9801442.

[215] L. Basso, S. Dittmaier, A. Huss, and L. Oggero, Techniques for the treatment of IR divergences in decay processes at NLO and application to the top-quark decay, arXiv:1507.0467.

[216] T. Ježo and P. Nason, On the Treatment of Resonances in Next-to-Leading Order Calculations Matched to a Parton Shower, arXiv:1509.0907. 
[217] T. Binoth, F. Boudjema, G. Dissertori, A. Lazopoulos, A. Denner, et al., A Proposal for a standard interface between Monte Carlo tools and one-loop programs, Comput.Phys.Commun. 181 (2010) 1612-1622, arXiv:1001.1307].

[218] S. Alioli, S. Badger, J. Bellm, B. Biedermann, F. Boudjema, et al., Update of the Binoth Les Houches Accord for a standard interface between Monte Carlo tools and one-loop programs, Comput.Phys.Commun. 185 (2014) 560-571, [arXiv:1308.3462].

[219] P. Nogueira, Automatic Feynman graph generation, J.Comput.Phys. 105 (1993) 279-289.

[220] J. Vermaseren, New features of FORM, math-ph/0010025.

[221] J. Kuipers, T. Ueda, J. Vermaseren, and J. Vollinga, FORM version 4.0, Comput.Phys.Commun. 184 (2013) 1453-1467, arXiv:1203.6543].

[222] G. Cullen, M. Koch-Janusz, and T. Reiter, Spinney: A Form Library for Helicity Spinors, Comput.Phys.Commun. 182 (2011) 2368-2387, arXiv:1008.0803.

[223] T. Reiter, Optimising Code Generation with haggies, Comput.Phys.Commun. 181 (2010) 1301-1331, arXiv:0907.3714.

[224] P. Mastrolia, E. Mirabella, and T. Peraro, Integrand reduction of one-loop scattering amplitudes through Laurent series expansion, JHEP 1206 (2012) 095, arXiv:1203.0291.

[225] H. van Deurzen, G. Luisoni, P. Mastrolia, E. Mirabella, G. Ossola, et al., Multi-leg One-loop Massive Amplitudes from Integrand Reduction via Laurent Expansion, JHEP 1403 (2014) 115, arXiv:1312.6678].

[226] T. Peraro, Ninja: Automated Integrand Reduction via Laurent Expansion for One-Loop Amplitudes, arXiv:1403.1229.

[227] P. Mastrolia, G. Ossola, C. Papadopoulos, and R. Pittau, Optimizing the Reduction of One-Loop Amplitudes, JHEP 0806 (2008) 030, arXiv:0803.3964].

[228] G. Ossola, C. G. Papadopoulos, and R. Pittau, On the Rational Terms of the one-loop amplitudes, JHEP 0805 (2008) 004, arXiv:0802.1876.

[229] G. Heinrich, G. Ossola, T. Reiter, and F. Tramontano, Tensorial Reconstruction at the Integrand Level, JHEP 1010 (2010) 105, arXiv:1008.2441.

[230] T. Binoth, J.-P. Guillet, G. Heinrich, E. Pilon, and T. Reiter, Golem95: A Numerical program to calculate one-loop tensor integrals with up to six external legs, Comput.Phys.Commun. 180 (2009) 2317-2330, arXiv:0810.0992]. 
[231] G. Cullen, J. P. Guillet, G. Heinrich, T. Kleinschmidt, E. Pilon, et al., Golem95C: A library for one-loop integrals with complex masses, Comput.Phys.Commun. 182 (2011) 2276-2284, arXiv:1101.5595.

[232] J. P. Guillet, G. Heinrich, and J. von Soden-Fraunhofen, Tools for NLO automation: extension of the golem95C integral library, Comput.Phys.Commun. 185 (2014) 1828-1834, arXiv:1312.3887.

[233] A. van Hameren, OneLOop: For the evaluation of one-loop scalar functions, Comput.Phys.Commun. 182 (2011) 2427-2438, [arXiv:1007.4716].

[234] T. Stelzer and W. Long, Automatic generation of tree level helicity amplitudes, Comput.Phys.Commun. 81 (1994) 357-371, hep-ph/9401258.

[235] J. Alwall, P. Demin, S. de Visscher, R. Frederix, M. Herquet, et al., MadGraph/MadEvent v4: The New Web Generation, JHEP 0709 (2007) 028, [arXiv:0706.2334].

[236] F. Maltoni and T. Stelzer, MadEvent: Automatic event generation with MadGraph, JHEP 0302 (2003) 027, hep-ph/0208156.

[237] M. Cacciari and G. P. Salam, Dispelling the $N^{3}$ myth for the $k_{t}$ jet-finder, Phys.Lett. B641 (2006) 57-61, hep-ph/0512210.

[238] M. Cacciari, G. P. Salam, and G. Soyez, The Anti-k(t) jet clustering algorithm, JHEP 0804 (2008) 063, arXiv:0802.1189.

[239] M. Cacciari, G. P. Salam, and G. Soyez, FastJet User Manual, Eur.Phys.J. C72 (2012) 1896, [arXiv:1111.6097.

[240] J. H. Kuhn, A. Kulesza, S. Pozzorini, and M. Schulze, Electroweak corrections to large transverse momentum production of $W$ bosons at the LHC, Phys.Lett. B651 (2007) 160-165, hep-ph/0703283.

[241] V. Bertone, S. Carrazza, D. Pagani, and M. Zaro, On the Impact of Lepton PDFs, arXiv:1508.0700,

[242] In Ref. [241] the lepton PDF has also been determined. It may be relevant, for example, in the computation of EW corrections to photon initiated processes.

[243] For example, in $e^{+} e^{-}$collisions the cancellation would require also the processes with $e^{+} \nu_{e}$, $e^{-\overline{\nu_{e}}}$ and $\nu_{e} \overline{\nu_{e}}$ as initial states, while in $p p$ collisions the cancellation would take place if $u$ and $d$ type quarks were weighted by the same PDFs. 
[244] Photon induced contributions have been included in Ref. [138].

[245] The Born-EW matrix elements squared do not enter directly our numerical computation, nevertheless these numbers might be useful to fix completely the setup. Furthermore, the relative amplitudes have to be computed anyway to build essential interference terms. 